\title{
Fear propagation and the evacuation dynamics
}

\author{
F.E. Cornes ${ }^{*, a}$, G.A. Frank ${ }^{c}$, C.O. Dorso ${ }^{\mathrm{a}, \mathrm{b}}$ \\ ${ }^{a}$ Departamento de Física, Facultad de Ciencias Exactas y Naturales, Universidad de Buenos Aires, Pabellón I, Ciudad Universitaria, Buenos Aires 1428, \\ Argentina \\ ${ }^{\mathrm{b}}$ Instituto de Física de Buenos Aires, Pabellón I, Ciudad Universitaria, Buenos Aires 1428, Argentina \\ ${ }^{\mathrm{c}}$ Unidad de Investigación y Desarrollo de las Ingenierías, Universidad Tecnológica Nacional, Facultad Regional Buenos Aires, Av. Medrano 951, \\ Buenos Aires 1179, Argentina
}

\section{A R T I C L E I N F O}

\section{PACS:}

45.70.Vn

89.65.Lm

\section{Keywords:}

Emergency evacuation

Social force model

Fear spreading

\begin{abstract}
A B S T R A C T
Fear may spread over a crowd in a similar fashion as contagious diseases do in social groups. People in a life-threatening situation may express fear alerting others of imminent danger. This social mechanism initiates an evacuation process, while affecting the way people try to escape. We developed a model that describe and reproduce the fear propagation mechanism, where many individuals may suddenly switch to an anxious state. We focused on video analyses in order to obtain reliable parameters from a real fear-propagation events, and further test these parameters on computer simulations. We observed that two evacuation schemes may appear, according to the pedestrians susceptibility. Both schemes exhibit different evacuation patterns and are qualitatively visible in the available real life recordings of crowded events. We quantified these patterns through topological parameters. We further investigated how the fear spreading gradually stops if the source of danger ceases.
\end{abstract}

\section{Introduction}

The evacuation processes attain different pedestrian behavioral patterns. Patient and impatient individuals, cooperation or competition, etc. are some commons behaviors that arise during emergency situations. Furthermore, the propagation of the pedestrians emotional states can be analyses through a convenient game theory model $[1,2]$. Our approach, however, will deal with behaviors during emergency situations, in the context of the social force model and the Susceptible-Infected-Recovered-Susceptible (SIRS) model.

Emergency situations may trigger different behavioral reactions. Some researches claim that "chaotic behavior" is not the only description of what happens during an emergency. They further consider other collective behaviors such as "cooperation" or "sociability", rather than individual's competition [3]. In this sense, people do not take course of actions that are solitary or selfpreservative only [4]. It has been notice that the shared experience of the same threat creates a sense of "we-ness" among the crowd. Therefore, a shared fate may create or even strengthen the social bonds. Through many interviews with survivors of emergencies, Ref. [3] reports that the identification with the others plays an important role during emergency situations.

Many video recordings from emergency situations shows pedestrians trying to escape from danger (see Section 3 for more details). We shall focus on this kind of reactions. The observed spreading of fear among this social groups implies some kind of coupling mechanism that exists between agents [5]. This coupling mechanism corresponds to social communication between the members of the group. As a consequence, it can be considered that the individuals (agents) may change their emotional state from the relaxed to

\footnotetext{
* Corresponding author. Tel.: +54011 43022149.

E-mail address: fercornes@gmail.com (F.E. Cornes).
} 
the fear one (and back again).

Fear propagation over the crowd can be attained if the coupling mechanism between individuals is strong enough and affects many neighboring pedestrians (meaning a high influence probability). Research on random lattices shows, however, that for a small number on neighbors (i.e. less than four) propagation is even possible if they exhibit really moving gestures [5].

Recent investigations suggests that other psychological mechanisms than social communication can play an important role during the fear spreading over the crowd [6-8]. Susceptibility, for example, appears as a relevant attribute that controls the fear propagation [6]. The Susceptible-Infected-Recovered-Susceptible (SIRS) model raises as a suitable research tool for examining the fear dynamics. The spreading model is, therefore, represented as a system of first order equations $[6,8]$.

According to the SIRS model implemented in Ref. [6], a dramatic spreading of fear can be expected in those crowded situations where the individuals are not able to calm down quickly. The speed at which the individuals calms down may not only depend on the current environment, but on other psychological attributes [6]. Ref. [9] proposes a characteristic value for this "stress decay".

Although the SIRS model appears to be a reasonable approach to fear spreading, it has been argued that it may not accurately resemble the situations of crowds with moving pedestrians [7,10]. The moving pedestrians will get anxious if their "inner stress" exceeds a threshold [10]. That is, if the cumulative emotions received by the pedestrian's neighbors surpasses a certain "inner stress" threshold.

Conversely, unlike the SIRS model, any anxious pedestrian may relax after some time due to "stress decay" (if no emotions of fear are received by the corresponding neighbors) $[7,10]$. That is, in this case, there is not a probability to switch from the anxious (infected) state to the relaxed (recovered) state as in the SIRS model, but a natural decay. Thus, the increase in the "inner stress" and the "stress decay" are actually the two main phenomena determining the pedestrians behavior.

Researchers seem not to agree on how the increase in the "inner stress" and the "stress decay" affect the pedestrians behavioral patterns [10-12]. Pelechano and co-workers [11] suggest that the maximum current velocity of the pedestrians may increase if he (she) gets anxious (i.e. with fear). But Fu and co-workers [10] propose to update the desired velocity (not the current one) of the pedestrian, according to his (her) current "inner stress" (see Section 2 for details). Both investigations assume that the pedestrians move in the context of the Social Force Model (SFM).

More experimental data needs to be examined before arriving to consensus on how the fear propagation affects the pedestrians dynamics.

Our investigation focuses on two real life situations. Our aim is to develop a model for describing the fear propagation mechanism, where many individuals may suddenly switch to an anxious state. We focused on video analyses in order to obtain reliable parameters from a real fear-propagation events, and further test these parameters on computer simulations.

We want to highlight that our approach attains fairly good agreement with the (analyzed) real life situations. Our main goal is that the way that fear spreads among the crowd depends on two major parameters, associated to the susceptibility to fear emotions and the later decay of this emotions. The fear propagation may exhibit different topological patterns according to the geometrical boundaries. We will detail these issues as follows.

In Section 2 we introduce the dynamic equations for evacuating pedestrians, in the context of the Social Force Model (SFM). We also define the meaning of the appearance to danger, the contagion stress and their relation to the pedestrians desired velocity.

In Section 3 we present the real life situations considered in our investigation. The corresponding simulations (in the SFM context) resembling these situations are detailed in Section 4.

Section 5 displays the results of our investigation, while some preliminary outcomes are summarized. Section 6 details the corresponding conclusions.

\section{Background}

\subsection{The social force model}

This investigation handles the pedestrians dynamics in the context of the "Social Force Model" (SFM) [13]. The SFM exploits the idea that human motion depends on the people's own desire to reach a certain destination at a given velocity, as well as other environmental factors [14]. The former is modeled by a force called the "desire force", while the latter is represented by "social forces" and "granular forces". These forces enter the equation of motion as follows

$$
m_{i} \frac{d \mathbf{v}^{(i)}}{d t}=\mathbf{f}_{d}^{(i)}+\sum_{j=1}^{N} \mathbf{f}_{s}^{(i j)}+\sum_{j=1}^{N} \mathbf{f}_{g}^{(i j)}
$$

where the $i, j$ subscripts correspond to any two pedestrians in the crowd. $\mathbf{v}^{(i)}(t)$ means the current velocity of the pedestrian $(i)$, while $\mathbf{f}_{d}$ and $\mathbf{f}_{s}$ correspond to the "desired force" and the "social force", respectively. $\mathbf{f}_{g}$ is the friction or granular force.

The $\mathbf{f}_{d}$ describes the pedestrians own desire to reach a specific target position at the desired velocity $v_{d}$. But, due to environmental factors (i.e. obstacles, visibility), he (she) actually moves at the current velocity $\mathbf{v}^{(i)}(t)$. Thus, he (she) will accelerate (or decelerate) to reach any desired velocity $v_{d}$ that will make him (her) feel more comfortable. Thus, in the Social Force Model, the desired force reads [13]

$$
\mathbf{f}_{d}^{(i)}(t)=m_{i} \frac{v_{d}^{(i)} \mathbf{e}_{d}^{(i)}(t)-\mathbf{v}^{(i)}(t)}{\tau}
$$


where $m_{i}$ is the mass of the pedestrian $i$ and $\tau$ represents the relaxation time needed to reach the desired velocity. $\mathbf{e}_{d}$ is the unit vector pointing to the target position. Detailed values for $m_{i}$ and $\tau$ can be found in Refs. [13,15,16].

The "social force" $\mathbf{f}_{s}(t)$ represents the socio-psychological tendency of the pedestrians to preserve their private sphere. The spatial preservation means that a repulsive feeling exists between two neighboring pedestrians, or, between the pedestrian and the walls $[13,14]$. This repulsive feeling becomes stronger as people get closer to each other (or to the walls). Thus, in the context of the Social Force Model, this tendency is expressed as

$$
\mathbf{f}_{s}^{(i j)}=A_{i} e^{\left(r_{i j}-d_{i j}\right) / B_{i}} \mathbf{n}_{i j}
$$

where ( $i j$ ) corresponds to any two pedestrians, or to the pedestrian-wall interaction. $A_{i}$ and $B_{i}$ are two fixed parameters (see Ref. [17]). The distance $r_{i j}=r_{i}+r_{j}$ is the sum of the pedestrians radius, while $d_{i j}$ is the distance between the center of mass of the pedestrians $i$ and $j . \mathbf{n}_{i j}$ means the unit vector in the $\overrightarrow{j i}$ direction. For the case of repulsive feelings with the walls, $d_{i j}$ corresponds to the shortest distance between the pedestrian and the wall, while $r_{i j}=r_{i}$ (see Refs. [13,14]).

It is worth mentioning that the Eq. (3) is also valid if two pedestrians are in contact (i.e. $r_{i j}>d_{i j}$ ), but its meaning is somehow different. In this case, $\mathbf{f}_{s}$ represents a body repulsion, as explained in Ref. [18].

The granular force $\mathbf{f}_{g}$ included in Eq. (1) corresponds to the sliding friction between pedestrians in contact, or, between pedestrians in contact with the walls. The expression for this force is

$$
\mathbf{f}_{g}^{(i j)}=\kappa\left(r_{i j}-d_{i j}\right) \Theta\left(r_{i j}-d_{i j}\right) \Delta \mathbf{v}^{(i j)} \cdot \mathbf{t}_{i j}
$$

where $\kappa$ is a fixed parameter. The function $\Theta\left(r_{i j}-d_{i j}\right)$ is zero when its argument is negative (that is, $\left.r_{i j}<d_{i j}\right)$ and equals unity for any other case (Heaviside function). $\Delta \mathbf{v}^{(i j)} \cdot \mathbf{t}_{i j}$ represents the difference between the tangential velocities of the sliding bodies (or between the individual and the walls). Detailed values for all the parameters $\left(m_{i}, \tau, A_{i}, B_{i}, \kappa\right)$ can be found in Ref. [13].

\subsection{The inner stress in the SFM context}

As mentioned in Section 1, the "inner stress" stands for the cumulative emotions that the pedestrian receives from his (her) neighbors. This magnitude may change the pedestrian's desired velocity (in the context of the Social Force Model). Relaxed pedestrians are expected to have low desired velocities, while anxious pedestrians (individuals with fear) attain a higher $v_{d}$. Following Ref. [10], we propose the inner stress $M(t)$ to be related with the desired velocity as

$$
v_{d}(t)=v_{d}^{\min }+\left(v_{d}^{\max }-v_{d}^{\min }\right) M(t)
$$

where $M(t)$ is assume to be bounded between zero (completely relaxed) and one (i.e. fear state). The minimum desired velocity $v_{d}^{\text {min }}$ corresponds to the (completely) relaxed state, while the maximum desired velocity $v_{d}^{\max }$ corresponds to the (completely) fear state (see Section 4 for details).

\subsection{The inner stress behavior}

The emotions received from the pedestrian's surrounding may switch his (her) inner stress $M(t)$ from the relaxed state (say, $M=0$ ) to the anxious state. However, in the absence of stressful situations, some kind of relaxation occurs (i.e., the "stress decay"), attaining a decrease in $M(t)$. Following Ref. [12], a first order approach for the time evolution of the "stress decay" reads as follows

$$
M(t)= \begin{cases}0 & \text { if relaxed } \\ \exp \left[-\left(t-t_{0}\right) / \tau_{M}\right] & \text { if anxious }\end{cases}
$$

It is assumed here that the pedestrian switched from the relaxed state to the anxious state at $t=t_{0}$. Thus, the stress decay process begins at $M=1$ and settles down with a characteristic time $\tau_{M}$. No further "contagion" mechanism is allowed during the "stress decay" process since the pedestrian is already in an anxious state.

Whenever the pedestrian receives alerting emotions from his (her) neighbors (say, at $t=t_{0}$ ), the "inner stress" is expected to increase. $\mathrm{He}$ (she) may switch to the anxious state if $k$ pedestrians among $n$ neighbors are expressing fear, as described in Appendix A. Fig. 1 shows the influencing area where the alerting (anxious) neighbors are supposed to be. This area is bounded by the contagion radius.

The contagion efficiency $\mathcal{P}$ is intended as the chance to mutate of state at the (fixed) "information time-steps" $\tau_{i}, 2 \tau_{i}, 3 \tau_{i}, \ldots$. The chance for this event to occur within the time interval $\left(t_{0}, t_{0}+d t\right)$ reads

$$
d \mathcal{P}(t)=\left\{\begin{array}{cl}
J\left\langle\frac{k}{n}\right\rangle \frac{d t}{\tau_{i}} & \text { if } m \tau_{i} \in\left(t_{0}, t_{0}+d t\right) \\
0 & \text { if not }
\end{array}\right.
$$

for $m=1,2,3, \ldots$. The transition rate of the (stochastic) process can be computed straight forward from the above, say, as $d \mathcal{P} / d t$. However, an appropriate formal expression for attaining the complete piecewise information in Eq. (7) should read as 


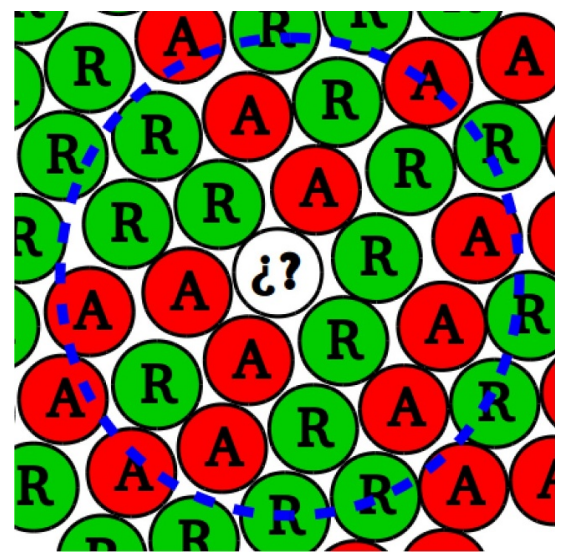

Fig. 1. Crowd with relaxed (green circles) and anxious (red circles) pedestrians. The state of the pedestrian labeled with question marks depends of the amount of neighboring anxious and relaxed individuals (see Eq. 7). The dashed blue circle indicate the contagious radius.

$$
\frac{d \mathcal{P}}{d t}=\int_{t_{0}}^{t_{0}+\tau} \frac{J}{\tau_{i}}\left\langle\frac{k}{n}\right\rangle \delta\left(t-m \tau_{i}\right) d t
$$

where $\delta(\cdot)$ stands for the Dirac's delta function. This function represents the fact that the pedestrians are allowed to mutate at fixed time-steps. Notice that $J / \tau_{i}$ corresponds to the contagion stress whenever the "information time-step" is set to $\tau_{i}$. We refer to this normalized contagion stress along the simulation processes.

The "imitation of neighbors" (say, $k$ among $n$ ) might not be the only way for transmitting fear. "Screams" and other sounds could enhance the information velocity along the crowd. However, our analysis of videos show that this "longer range" propagation has low probability to occur (see Section 3 for details). We therefore assumed a (long range) vanishing probability in our model.

The parameter $J$ represents an effective contagion stress (see Appendix A for details). This parameter resembles the pedestrian susceptibility to get anxious (i.e. with fear). That is, it links the propagation efficiency $\mathcal{P}$ (effects of received emotions) with the mean number of neighbors expressing fear. For simplicity, we assume that this parameter is the same for all the pedestrians. In Appendix B we estimated an experimental value of the effective contagion stress $J$ for the Turin incident. Finally, we want to remark that the term "contagion stress" should not be associated to any former term appearing in the literature; its meaning is intended to be restricted to the context of this work.

Notice that vanishing values of $J$ will not affect the emotional state of the pedestrian (not increase his (her) inner stress). Nonvanishing values of $J$, on the contrary, will increase the tendency of the pedestrian to switch to an anxious state. As mention in Appendix A, $J$ is intended as an "effective" parameter including the statistics of the alerting neighbors.

Finally, we want to remark that the increase or decay of the inner stress $M$ does not correspond to any psychological theory. We should also emphasize that, from our point of view, both behaviors rely on what we observed through the recordings of the incidents detail in Section 3.

\subsection{The $\tau_{M}$ determination}

Eqs. (5) and (6) proposes the way to handle an estimation of $\tau_{M}$. Whenever the desired velocity $v_{d}(t)$ is known at $t=t_{c}$, the characteristic time $\tau_{M}$ may be expressed as follows (assuming $t_{0}=0$ )

$$
\tau_{M}^{-1}=\frac{1}{t_{c}} \ln \left(\frac{v_{d}^{\max }-v_{d}^{\min }}{v_{d}\left(t_{c}\right)-v_{d}^{\min }}\right)
$$

Ref. [9] suggests that $\tau_{M} \simeq 50$ seconds. We will show in Section 4.2 that this value appears as a somewhat upper limit for $\tau_{M}$. The above expression, however, undergoes to values approaching $10 \mathrm{~s}$ (or lower), as we will explain in Section 4.

\subsection{Topological characterization of the escaping pattern}

One of the most useful image processing technique is the computation of the Minkowski functionals. This general method, based on the concept of integral geometry, uses topological and geometrical descriptors to characterize the topology of two and three dimensional patterns [19].

We used this method to analyze data (images) obtained from the video of the Charlottesville incident. So, we focused the attention on the 2-D case. Three image functionals can actually be defined in 2-D: area, perimeter and the Euler characteristic. The three can give a complete description of 2-D topological patterns appearing in (pixelized) black and white images.

To characterize a pattern on a black and white image, each black (or white) pixel is decomposed into 4 edges, 4 vertices and the interior of the pixel or square. Taking into account the total number of squares $\left(n_{s}\right)$, edges $\left(n_{e}\right)$ and vertices $\left(n_{v}\right)$, the area $(A)$, 


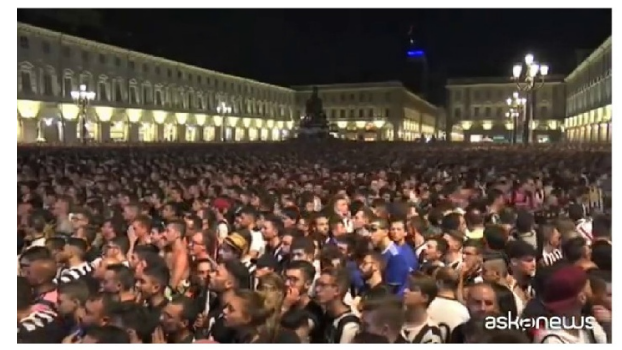

(a) Pedestrians watching the screen.



(b) Pedestrians with fear.

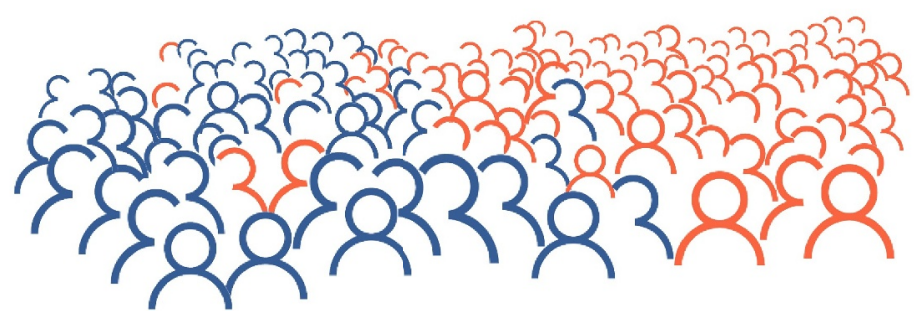

(c) Fear spreading.

Fig. 2. (a) Snapshot of the crowd watching the football match. The screen is on the left (out of the scene). The pedestrians on the right are actually anxious (with fear) due to the fake bomber. The (b) snapshot corresponds to the same scene as (a) but shifted to the state $10 \mathrm{~m}$ right (actually, the camera appearing in this image is the one that captured the (a) image). The fake bomber appears in the scene and is indicated with a green arrow. (c) Analysis of the fear spreading among the crowd. The blue and orange profiles represent the relaxed and anxious pedestrians, respectively, associated to the (a) image (see text for details). The total number of contour bodies is $N=131$.

perimeter $(U)$ and Euler characteristic $(\chi)$ are defined as

$$
A=n_{s}, \quad U=-4 n_{s}+2 n_{e}, \quad \chi=n_{s}-n_{e}+n_{v}
$$

The area is simply the total number of (black or white) pixels. The second and third Minkowski functionals describe the boundary length and the connectivity or topology of the pattern, respectively. The latter corresponds to the number of surfaces of connected black (white) pixels minus the number of completely enclosed surfaces of white (black) pixels (see Ref. [20]).

\section{Experimental data}

In this section we introduce two incidents, as examples of real life fear propagation. The first one occurred in Turin (Italy) while the other one took place in Charlottesville (USA), both in 2017. We further present relevant data extracted from the corresponding videos available in the web (see on-line complementary material).

\subsection{Turin (italy)}

On June 3rd 2017, many Juventus fans were watching the Champions League final between Juventus and Real Madrid on huge screens at Piazza San Carlo. During the second half of the match, a stampede occurred when one (or more) individuals shouted that there was a bomb. More than 1000 individuals were injured during the stampede, although it was a false alarm. Fig. 2 captures two moments of the fear spreading (see caption for details). The arrow in Fig. $2 b$ points to the individual that caused the fear spreading. He will be called the fake bomber throughout this investigation.

The recordings from Piazza San Carlo show how the pedestrians escape away from the "fear source", that is, from the fake bomber. It can be seen in Fig. $2 \mathrm{~b}$ the opening around the fear source a few seconds after the shout. The opening exhibits a circular pattern around the fake bomber. This behavior gradually slows down as the pedestrians realize the alarm being false. Approximately 20 seconds after the shout, the pedestrians calms down to the relaxed state while the opening closes.

In order to quantify the fear propagation among the crowd, we split the video into 14 images. The frame rate was 2 frames per second. Thus, the time interval between successive images was 0.5 seconds.

Fig. 2c shows the profile corresponding to the Fig. 2a. Any (distinguishable) pedestrian in Fig. 2a is outlined in Fig. 2c as a body contour. The contour colors represent the state of the pedestrians, relaxed pedestrians are outlined in blue (on-line version) and anxious pedestrians are outlined in orange (on-line version). The latter correspond to the individuals that suddenly changed their motion pattern. That is, individuals that turned back to see what happened or pedestrians that were pushed towards the screen (on the left) due to the movement of his (her) neighbors.

The fear spreading shown in Fig. 2c occurs from right to left, until nearly all the contours bodies switch to the "fear" state (i.e. orange in the on-line version). Notice, however, that a few pedestrians may remain relaxed for a while, even though his (her) 


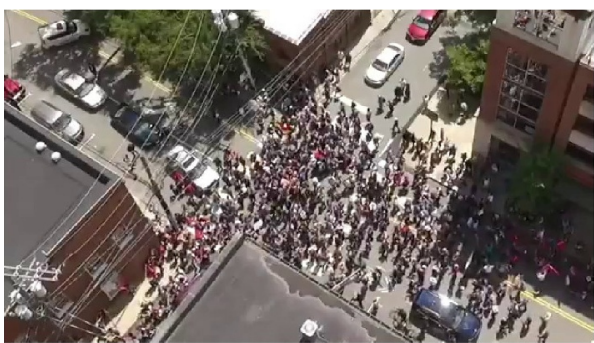

(a) Original frame.

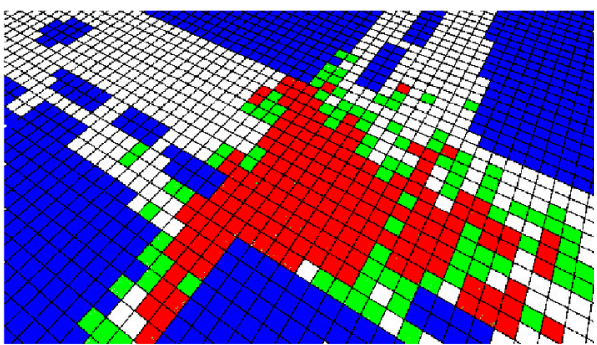

(b) Pixelated image.



(c) Perspective correction.

Fig. 3. (a) Image of the incident in Charlottesville. This image corresponds to the first frame of the video. (b) Pixelated image of the original frame. In the image we identify in blue color the obstacles, like cars and buildings. Green and red cells are occupy by one and more than one pedestrians, respectively. The street was colored in white. The line spacing was 12 pixels. The cell size was, approximately, $1.5 \mathrm{~m} \times 1.5 \mathrm{~m}$. (c) Perspective correction of the pixelated image. In green color we represents the position of the occupied cells by pedestrians. The white spaces represents the obstacles (buildings and cars) and the street.

neighbors have already switched to the fear state. Or, on the contrary, pedestrians with fear may be completely surrounded by relaxed pedestrians, as appearing on the left of Fig. 2c. Both instances are in agreement with the hypothesis that pedestrians may switch to a fear state according to a propagation efficiency $\mathcal{P}$. See Appendix A for details on the $\mathcal{P}$ computation within the "contagion" radius.

The inspection of successive images provides information on the new anxious pedestrians and the state of their current neighbors. Appendix B summarizes this information, while detailed values for the propagation efficiency $\mathcal{P}$ and the contagion stress $J$ are reported in Table B.2. Notice that the data sampling is strongly limited by the total number of outlined pedestrians (that is, 131 individuals). Thus, the reported values for $t>4 \mathrm{~s}$ are not really suitable as parameter estimates because of the finite size effects. In order to minimize the size effects, we focused on the early stage of the propagation where the contagion stress $J$ seems to be (almost) stationary (see Fig. B.15).

The (mean) contagion stress for the Turin incident was found to be $J=0.1 \pm 0.055$, within the standard deviation (see Appendix B for more details). This value appears to be surprisingly low according to explored values in the literature (see Ref. [12]). However, we shall see in Section 5 that this stress is enough to reproduce this real incident.

\subsection{Charlottesville, virginia (USA)}

One person was killed and 19 injured when a car ran into a crowd of pedestrians during an antifascist protest (Charlottesville, August 12th, 2017). The incident took place at the crossing of Fourth St. and Water St. Fig. 3a shows a snapshot of the incident (the video is provided in the supplementary material).

In the video, we can see that the whole crowd gets anxious. But, we can identify two groups of pedestrians, according to the amount of information they have about the incident. The individuals near the car (say, less than 5 m) actually witnessed when the driver ran over into the crowd. However, far away pedestrians become aware that something happened among the crowd due to the fear emotions of their neighbors. But, they cannot determine the real nature of the incident because the car is out of their sight. Thus, the pedestrians nearer to the car have more information than the far away individuals.

The video also shows that the pedestrians close to the car stop running as soon as the car stops. On the contrary, individuals located far away continue escaping after this occurs due to their lack of information.

Using the program ImageJ [21], we followed the trajectories of various pedestrians. Each trajectory corresponds to the joint of 19 points. Each one corresponds to the position of the same pedestrian over each frame. When the last point was indicate, the program automatically draw the trajectory of the respectively pedestrian over the last frame. As shown in Fig. 4, most of the trajectories are approximately radial to the car. Notice, however, that three individuals ran toward the car to help the other injured pedestrians.

In order to obtain more experimental data, we split the video into 19 frames. The frame rate was two frames per second. We 


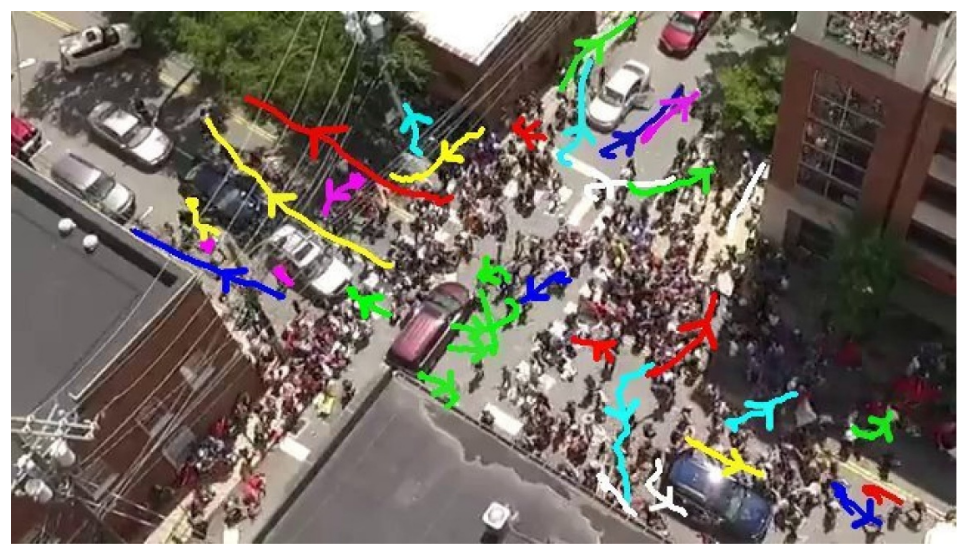

Fig. 4. Trajectories for some anxious pedestrians from the Charlottesville video. The pedestrians' positions were recorded on consecutive images, and then joined by means of the software ImageJ. The arrows represent the movement direction.

further overlapped a square grid on each frame, but taking into account the two-point perspective of each image. Each cell was colored with different colors depending if it was occupied by pedestrians, obstacles, etc (see caption in Fig. $3 \mathrm{~b}$ for details). Finally, we performed a back-correction of the perspective for a better inspection of the grid. The result is shown in Fig. 3c.

The complete analysis of the geometrical and topological patterns appearing on the grid can be found in Section 5 .

\section{Numerical simulations}

\subsection{The simulation conditions}

\section{The Turin scenario}

We mimicked the Turin incident (see Section 3) by first placing 925 pedestrians inside a $21 \mathrm{~m} \times 21 \mathrm{~m}$ square region. The pedestrians were placed in a regular square arrangement, meaning that the occupancy density was approximately 2 people $/ \mathrm{m}^{2}$. After their desire force was set (see below), the crowd was allowed to move freely until the system reaches the balance state before initiating the simulation. This balance situation can be seen in Fig. 5a and corresponds to the initial configuration for the fear spreading simulation.

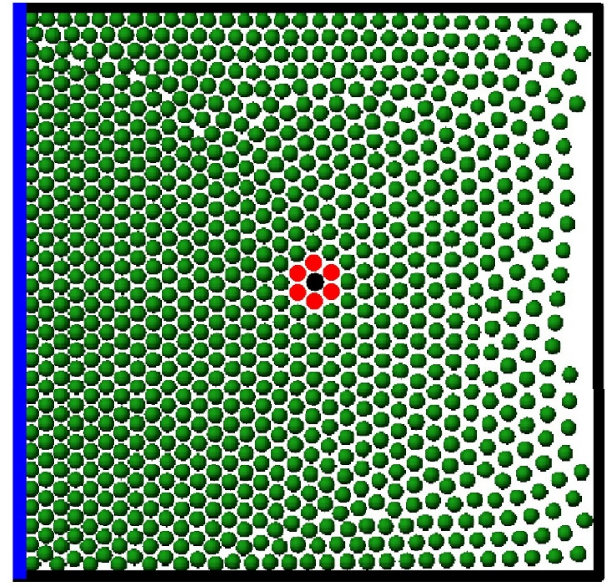

(a) Turin initial state.



(b) Charlottesville initial state.

Fig. 5. Snapshots of the initial configuration for the (a) Turin (Italy) and (b) Charlottesville (Virginia, USA) simulations. The relaxed pedestrians are represented in green circles on both images. (a) The fake bomber is represented in black, while his first neighbors are represented in red (see text for details). The blue line on the left represents the wide screen. (b) The car is represented by 21 black circles and moves from bottom to top, while its first neighbors are represented in red (see text for details). The solid (black) lines represent the walls and other obstacles (i.e. parked cars on the middle left of the image (see Fig. 3)). The width of the street at the bottom of the image is $8 \mathrm{~m}$ and at the top reaches $15 \mathrm{~m}$ (see red dashed lines). The street width on the left is divided into two paths of $5 \mathrm{~m}$ each, while the street width on the right is $13 \mathrm{~m}$ (see red dashed lines). Initially, the pedestrians were placed at random positions inside the region bounded by the red dashed lines. 
The on-line blue line on the left of Fig. 5a represents the wide screen mentioned in Section 3.1. We assumed that the pedestrians are attracted to the screen in order to have a better view of the football match. Thus, a (small) desire force pointing towards the screen was included at the beginning of the simulation. This force equaled $m v_{d} / \tau$ for the standing still individuals $(v(0)=0)$, according to Eq. (2). We further assumed that the pedestrians were in a relaxed state at the beginning of the simulation, and therefore, we set $v_{d}=0.5 \mathrm{~m} / \mathrm{s}$ [17]. This value accomplished a local density that did not exceed the maximum expected for outdoor events, say, $3-4$ people $/ \mathrm{m}^{2}[25]$.

The pedestrian in black in the middle of the crowd in Fig. 5a represents the fake bomber appearing in the video. He is responsible for triggering the fear propagation at the beginning of the simulations. For simplicity, we assume that he remain still during the fear spreading process.

The pedestrians in red in Fig. 5a are responsible for triggering the alert, as they are very close to the fake bomber (less than $1 \mathrm{~m}$ ). They were initially set to the fear state in the simulation (see Section 4.2 for details).

Recall that the event takes place outdoor. Piazza San Carlo, however, is surrounded by walls (as can be seen in Fig. 2a). Thus, we considered along the simulations that the crowd always remained inside the piazza and no other pedestrian were allowed to get inside during the process.

\section{The Charlottesville scenario}

The initial conditions for simulating the incident at Charlottesville are somewhat different from those detailed in Section 4.1. The pedestrians are now placed at random positions within certain limits around the street crossing (see Fig. 5b). But, in order to counterbalance the social repulsion between pedestrians, a (small) inbound desire force was set. That is, the pedestrian's desire force pointed to the center of the crossing.

The total number of pedestrians appearing in Fig. $5 \mathrm{~b}$ is 600 . This number was computed taking into account the total number of occupied cells and the amount of pedestrians per cell of the first frame of the video (see caption of Fig. 3b for details). Due to the low image quality of Fig. 3a, we were not able to distinguish if more than two pedestrians occupy a given cell. Thus, we simply assumed that each red cell was occupied by only two pedestrians.

After setting the pedestrian's desire velocity to $v_{d}=0.5 \mathrm{~m} / \mathrm{s}$, we allowed them to move freely towards the center of the street crossing. This instance continued until a similar profile to the one in Fig. 3a was attained.

Then, we assumed, according to the video, that the pedestrians tried to stay at a fix position. Thus, we set the desired velocity to zero and allowed the system to reach the balance state before initiating the simulation. Notice that this condition differed slightly from the Turin condition, where the desired velocity was set to $v_{d}=0.5 \mathrm{~m} / \mathrm{s}$ due to his (her) own desire to reach the screen (see Section 4.1).

The "source of fear" for the Charlottesville's incident corresponds to the offending driver moving along the y direction in Fig. 5b. We modeled the offending driver as a packed group of 21 spheres, (roughly) emulating the contour of a car (see Fig. 5b). The mass of the packed group was set to $2000 \mathrm{~kg}$. The car moved along the y direction at $3 \mathrm{~m} / \mathrm{s}$ until it reached the center of the street intersection. When this occurred, it stopped and remained fix until the end of the simulation.

As in the Turin situation, we assumed that those pedestrians very close to the car (that is, less than $1 \mathrm{~m}$ ) gets anxious immediately, and thus, they were initially set to the fear state (red circles in Fig. 5b). When the car stopped, the "source of fear" was switched off.

The streets are considered as open boundary conditions. This means that the pedestrians are able to rush away from the crossing as far as they can, unlike the Turin situation.

\subsection{The simulation process}

The videos that capture the fear spreading over the crowd let us classify the pedestrians into those moving relaxed or those moving anxiously. The former move somewhat loosely, while the later try to escape from the source of fear. Both patterns can be easily recognized through the videos. Indeed, we identified the different pedestrian behaviors through the simple observation of the recordings. In other words, we do not used a software in order to classify each behavior type.

An accurate determination of the inner stress $M$ seems not to be possible from the videos. Thus, we assume that the pedestrians may be in one of two possible states: relaxed or with fear. The former means that his (her) desired velocity does not exceed a fix threshold $v_{d}^{\lim }$, according to Eq. (5). The latter means that the individual surpassed this threshold (in the context of the Social Force Model).

We already mentioned in Section 4.1 that the desired velocity $v_{d}=0.5 \mathrm{~m} / \mathrm{s}$ is in correspondence with an accepted literature value for relaxed individuals. Hence, we set $v_{d}^{\text {lim }}=0.5 \mathrm{~m} / \mathrm{s}$ as a reasonable limit for the pedestrian to be considered relaxed. This limit is supposed to be valid for either the Turin and the Charlottesville incident, since the total number of pedestrians involved in each event and the expected maximum local density are similar in both cases.

For simplicity, $v_{d}^{\min }$ and $v_{d}^{\max }$ (Eq. (5)) were set to zero and $4 \mathrm{~m} / \mathrm{s}$, respectively, in all the simulations (Turin and Charlottesville). The maximum desired velocity $v_{d}^{\max }=4 \mathrm{~m} / \mathrm{s}$ corresponds to reasonable anxiety situations appearing in the literature [14,15,22,23].

Fig. 6 illustrates the time evolution for the desired velocity $v_{d}(t)$ of an individual who switches from the relaxed state to the fear state (see caption for details). Notice that the increase in the inner stress is implemented as an (almost) instantaneous change in his (her) desired velocity due to fear propagation. Once anxious, however, the stress decay phenomenon applies, regardless of any other neighbor expressing fear (see Section 2.3). The stress decay stops when the individual settles to the relaxed state, that is, when the $v_{d}$ returns back to $0.5 \mathrm{~m} / \mathrm{s}$. When this occurs, the pedestrian moves randomly at this desired velocity until the end of the simulation.

Notice that either in the Turin and the Charlottesville situation (see Figs. 2b and 4), the anxious pedestrians escapes radially from 


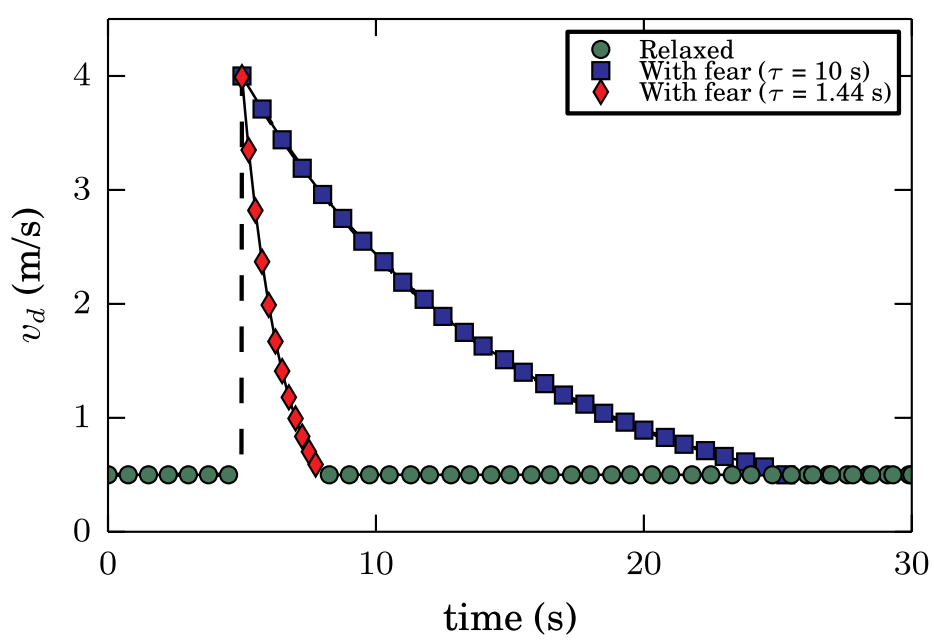

Fig. 6. Time evolution of the desired velocity $v_{d}$ for a single individual. The green color corresponds to the relaxed state, while the red and blue colors correspond to the fear state. At $t=5 \mathrm{~s}$ the $v_{d}$ switches from $0.5 \mathrm{~m} / \mathrm{s}$ to $4 \mathrm{~m} / \mathrm{s}$ due to fear propagation. No further stimulus is received after the spreading. The desired velocity decays exponentially from $t>5 \mathrm{~s}$ resembling the stress decay (Eq. (6) with $t_{0}=5 \mathrm{~s}$ ). The red diamond and blue square symbols represents two exponential characteristic times: $\tau_{M}=1.44 \mathrm{~s}$ and $\tau_{M}=10 \mathrm{~s}$, respectively. At $t=8 \mathrm{~s}$ and $t=25 \mathrm{~s}$ (depending the value of $\tau_{M}$ ) the desired velocity attains $v_{d}^{\lim }=0.5 \mathrm{~m} / \mathrm{s}$ and the individual returns to the relaxed state.

the source of fear. That is, from the fake bomber or the offending driver.

In order to determine the experimental value of the characteristic time $\tau_{M}$, we measured the time required by an anxious pedestrian to recover his (her) relaxed state $\left(t_{c}\right)$. According to the analysis of the videos, in the Turin's case, anxious pedestrians return to a relaxed state after $t_{c}=20$ seconds.

But, in the Charlottesville case, pedestrians near to the car (less than $5 \mathrm{~m}$ ) relaxed after $t_{c}=3$ seconds. However, far away pedestrians arrive to the relaxed state after $t_{c}=20$ seconds. Recall from Section 3.2, that individuals close to the car has more information about the incident nature than far away ones. Thus, when the car stops, near individuals recover his relaxed state, unlike to the far away pedestrians that remain anxious.

We computed the experimental value of the characteristic time $\tau_{M}$ for each scenario using Eq. (9) with $v_{d}^{\min }=0 \mathrm{~m} / \mathrm{s}, v_{d}^{\max }=4 \mathrm{~m} / \mathrm{s}$ and $v_{d}\left(t_{c}\right)=0.5 \mathrm{~m} / \mathrm{s}$. In the Turin's case, $\tau_{M}$ equals, approximately, to 10 seconds, while in the Charlottesville's case the characteristic time equals to 1.44 seconds and 10 seconds for pedestrians located close to the car or far away from it, respectively.

Notice that an anxious pedestrian has more or less "influence" over his neighbors according his (her) information level about the incident. That is, for example an individual near to the car can spread his (her) fear emotion over a relaxed pedestrian during 3 seconds only. In other words, during the time that the pedestrians were in the fear state $\left(t_{c}\right)$.

Finally, Table 1 summarizes the most usual values for the experimental parameters appearing in Eqs. (1)-(9).

Table 1

Most relevant parameters used for simulating both incidents.

\begin{tabular}{|c|c|c|c|c|}
\hline Magnitude & Symbol & Value & Units & Obtained in \\
\hline Pedestrian mass & $m_{i}$ & 70 & $\mathrm{~kg}$ & Ref. [13] \\
\hline Pedestrian radius & $r_{i}$ & 0.3 & $\mathrm{~m}$ & Ref. [13] \\
\hline Acceleration time & $\tau$ & 0.5 & $\mathrm{~s}$ & Ref. [13] \\
\hline Social force at $d_{i j}=r_{i j}$ & $A_{i}$ & 2000 & $\mathrm{~N}$ & Ref. [13] \\
\hline Characteristic length & $B_{i}$ & 0.08 & $\mathrm{~m}$ & Ref. [13] \\
\hline Contact distance & $r_{i j}$ & 0.6 & $\mathrm{~m}$ & Ref. [13] \\
\hline Friction coefficient & $\kappa$ & $2.4 \times 10^{5}$ & $\mathrm{~kg} \mathrm{~m}^{-1} \mathrm{~s}^{-1}$ & Ref. [13] \\
\hline Minimum desired velocity & $v_{d}^{\min }$ & 0.0 & $\mathrm{~m} / \mathrm{s}$ & Set by authors \\
\hline Maximum desired velocity & $v_{d}^{\max }$ & 4.0 & $\mathrm{~m} / \mathrm{s}$ & Set by authors \\
\hline Relaxed desired velocity & $v_{d}\left(t_{c}\right)$ & 0.5 & $\mathrm{~m} / \mathrm{s}$ & Set by authors \\
\hline $\begin{array}{l}\text { Relaxation time } \\
\text { (Turin, Charlottesville - far away) }\end{array}$ & $t_{c}$ & 20 & $\mathrm{~s}$ & Video analysis \\
\hline $\begin{array}{l}\text { Relaxation time } \\
\text { (Charlottesville - near) }\end{array}$ & $t_{c}$ & 3.0 & s & Video analysis \\
\hline $\begin{array}{l}\text { Characteristic time } \\
\text { (Turin, Charlottesville - far away) }\end{array}$ & $\tau_{M}$ & 10.0 & $\mathrm{~s}$ & Eq. (9) \\
\hline $\begin{array}{l}\text { Characteristic time } \\
\text { (Charlottesville - near) }\end{array}$ & $\tau_{M}$ & 1.44 & $\mathrm{~s}$ & Eq. (9) \\
\hline
\end{tabular}




\section{The fear propagation process}

The fear propagation process was implemented as follows. First, we associated an effective contagion stress $\mathcal{P}^{(i)}$ to each relaxed individual (at instance $i$ ). This magnitude corresponds to the computational approach for Eq. (7) by setting $d t \sim \tau_{i}$. Therefore, the computational contagion stress reads as follows

$$
\mathcal{P}^{(i)}=J\left\langle\frac{k}{n}\right\rangle
$$

The fractional of alerting neighbors $k / n$ in the above expression are those within a fix "contagion" radius of $2 \mathrm{~m}$.

Secondly, we switched the state of each relaxed individual to the fear state with chance $\boldsymbol{P}^{(i)}$. We introduced here a stochastic process in the "contagion" propagation. The process was repeated for successive instances $i+1, i+2$, etc. The time-step between instances was $\tau_{i}=0.05 \mathrm{~s}$ as explained below. Further details can be found in Appendix C.

Although the dynamics of the whole crowd was updated every $10^{-4} \mathrm{~s}$ (see below), the relaxation time of the pedestrians corresponds to $\tau=0.5 \mathrm{~s}$. Thus, there was no need to update each desired velocity $v_{d}(t)$ at periods shorter than, for example, a tenth of $\tau$ (that is, $0.05 \mathrm{~s}$ ). Consequently we set the "information time-step" (instances) as $0.05 \mathrm{~s}$. This means that the modulus of $v_{d}$ remained unchanged for at least $0.05 \mathrm{~s}$ (although its direction $\hat{\mathbf{e}}_{d}$ could change).

Notice that this spreading process may be envisaged as a Susceptible-Infected-Susceptible (SIS) process. The Susceptible-toInfected transit corresponds to the (almost immediate) increase of $v_{d}$ from $0.5 \mathrm{~m} / \mathrm{s}$ to $4 \mathrm{~m} / \mathrm{s}$ (with effective contagion stress $\mathcal{P}^{(i)}$ ). The Infected-to-Susceptible transit corresponds to the stress decay from $4 \mathrm{~m} / \mathrm{s}$ back to $0.5 \mathrm{~m} / \mathrm{s}$.

We want to remark again the fact that the emotions received by an individual in the fear state were neglected, and thus, did not affect the stress decay process. This should be considered as a first order approach to the fear propagation process.

\section{The stress decay process}

The stress decay process applies to the group of pedestrians with fear (i.e. anxious state in Eq. (6). As mentioned previously, any emotional stimulus on this group is disregarded as long as the individual belongs to this group (that is, he (she) remains in the anxious state). Recall that this state lasts until his (her) desired velocity settles down to $0.5 \mathrm{~m} / \mathrm{s}$.

The $v_{d}(t)$ updates (every $0.05 \mathrm{~s}$ ) were done according to Eq. (5). The corresponding values of $v_{d}^{\min }$, $v_{d}^{\max }$ and $\tau_{M}$ were previously computed from experimental data, as explained above. Recall that a single value of $\tau_{M}$ could be established for the Turin situation, but two values were required for the Charlottesville situation, due to the pedestrians' proximity to the source of danger (see above).

Whenever the pedestrian's $v_{d}$ drops off $0.5 \mathrm{~m} / \mathrm{s}$, he (she) is no longer considered as an anxious individual, and thus, his (her) stress decay process ceases. Eq. (5) does not further apply to this individual, but he (she) becomes again susceptible to fear spreading. The increase of his (her) inner stress occurs as described in the previous Section.

\section{Simulation software}

The simulations were implemented on the LAMMPS molecular dynamics simulator [24]. LAMmPs was set to run on multiple processors. The chosen time integration scheme was the velocity Verlet algorithm with a time step of $10^{-4} \mathrm{~s}$. Any other parameter was the same as in previous works (see Refs. [15,22]).

We implemented special modules in $\mathrm{C}++$ for upgrading the LAmmps capabilities to attain the "social force model" simulations. We performed between 60 and 90 simulations for each situation (see figures caption for details). Also, the processes lasted between $10 \mathrm{~s}$ and $20 \mathrm{~s}$ according each analysis. Data was recorded at time intervals of $0.05 \mathrm{~s}$ and $0.005 \mathrm{~s}$. The recorded magnitudes were the pedestrian's positions and their emotional state (relaxed or anxious) for each evacuation process.

\section{Results}

In this section we discuss the results obtained from either real life situations and computer simulations. Two sections enclose these results in order to discuss them in the right context. We first analyze the Turin case (Section 5.1), while the more complex one (Charlottesville, Virginia) is left to Section 5.2.

\subsection{Turin}

\subsubsection{The contagion stress parameter}

As a first step, we measured the mean number of pedestrians that switched to anxious in the first $20 \mathrm{~s}$ of the escaping process for a wide range of contagion stresses $(J)$. This is shown in Fig. 7. As can be seen, the number of anxious pedestrians increases for increasing contagion stresses. That is, as pedestrians become more susceptible to the fear emotions from his (her) neighbors, more pedestrians gets anxious.

The fraction of pedestrians that switch to the anxious state can be classified into three qualitative categories as shown in Fig. 7. For $J$ ranging between 0 to 0.01 , no significant spreading appears. But this scenario changes rapidly for the $J$ (intermediate) range between 0.01 and 0.03 . The slope in Fig. 7 displays a maximum throughout this interval. However, if the stress becomes stronger (say, above 0.03), the majority of the crowd enters into an anxious state regardless of the precise value of $J$. A threshold for this is around $J=0.04$.

Notice that Fig. 7 is in agreement with the experimental Turin value for the mean contagion stress $(J=0.100 \pm 0.055$, see 


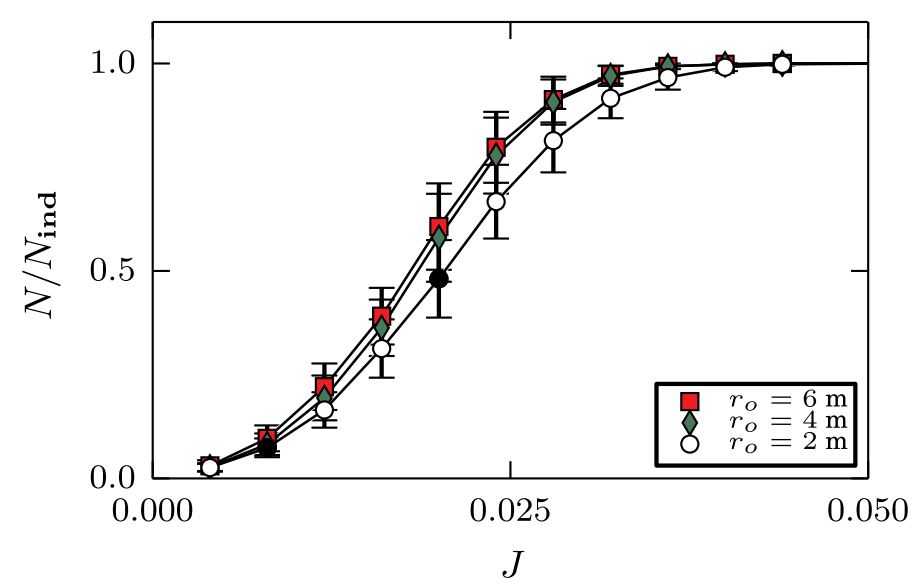

Fig. 7. Normalized number of pedestrians that switched to anxious in the first $20 \mathrm{~s}$ of the escaping process as a function of the contagion stress $J$ for contagious radius $r_{o}=2 \mathrm{~m}, 4 \mathrm{~m}$ and $6 \mathrm{~m} . N$ is the number of anxious pedestrians. The plot is normalized with respect to the total number of individuals $\left(N_{\text {ind }}=925\right)$. $J$-values of 0.01 and 0.02 are indicated in black color (and circle symbols). Mean values were computed from 60 realizations. The error bars corresponds to $\pm \sigma$ (one standard deviation).

Section 3). The fear situation at Piazza San Carlo, as observed from the videos, shows that all the pedestrians moved to the fear state. The snapshot in Fig. 2b illustrates the situation a while after the (fake) bomber called for attention.

The fear spreading shown in Fig. 7 does not appear to change significantly for increasing "contagion" radii. We explored situations enclosing only first neighbors $(2 \mathrm{~m}$ ) to situations enclosing as far as $6 \mathrm{~m}$. Notice that $J=0.025$ seems to be an upper limit for any weak fear spreading situation, or the lower limit for any widely spreading situation.

Following the above working hypothesis, we turned to study any morphological evidence for both regimes in Section 5.1.2.

\subsubsection{The morphology of the escaping pattern}

Our next step was to examine the anxious pedestrian's spatial distribution for the Piazza San Carlo scenario. The corresponding videos show that the individuals tried to escape radially from the (fake) bomber (see Fig. 2b). Thus, the polar space binning (i.e. cake slices) centered at the (fake) bomber seemed the most suitable framework for inspecting the crowd morphology by pieces. We binned the piazza into $N_{\text {bins }}=30$ equally spaced pieces as shown in Fig. $5 \mathrm{a}$. The angle between consecutive bins was $\varphi=360^{\circ} / N_{\text {bins }}=12^{\circ}$.

Fig. 8 exhibits the number of bins or slices (normalized by the total number of bins) occupied at least by one anxious pedestrian. Three different spreading situations are represented there. These situations represent the qualitative categories mentioned in Section 5.1.1. That is, $J=0.01$ for low fear spreading, $J=0.02$ for an intermediate spreading and $J=0.09$ for wide fear spreading (see caption of Fig. 8 for details).

According to Fig. 8, the number of occupied bins (slices) increases monotonically during the escaping process. This means that fear propagates in all directions (from the bomber) until nearly all the slices become occupied. However, the slopes in Fig. 8 for each


(a)

(b)

Fig. 8. (a) Schematic representation of the radial (left) and circular (right) bins (see text for more details). The red circles represent the position of many anxious pedestrians. The fake bomber is placed at the center of the region. (b) Fraction of occupied radial bins by anxious pedestrians $v s$. time (in seconds) for $J=0.01,0.02$ and $0.09 . N$ is the number of occupied radial bins (see text for more details). The plot is normalized with respect to the total number of radial bins $\left(N_{\text {bins }}=30\right)$. The contagious radius was $2 \mathrm{~m}$. Mean values were computed from 60 realizations. The error bars corresponds to $\pm \sigma$ (one standard deviation). 


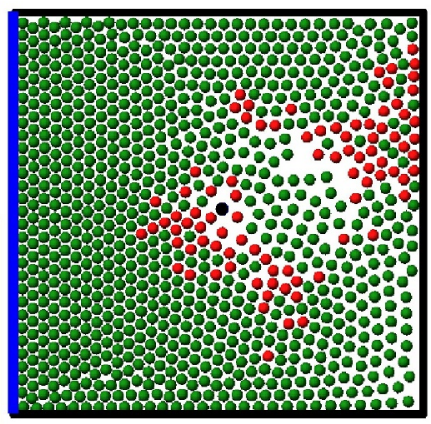

(a) $J=0.01$

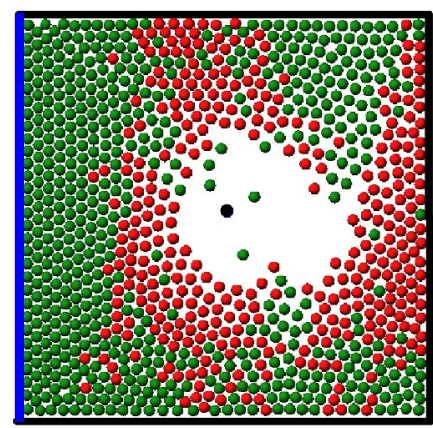

(b) $J=0.02$

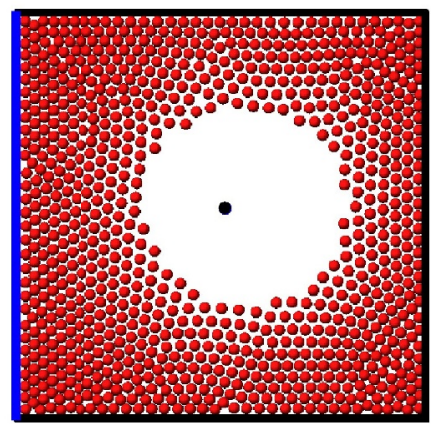

(c) $J=0.09$

Fig. 9. Snapshots of different escaping processes for three values of contagion stress in the first 15 seconds. The different colors of the circles represents the anxiety state of each pedestrian. Relaxed and anxious pedestrians are represent in green and red circles, respectively. The fake bomber is place at the center of the region and is represented in black circle. Relaxed pedestrians desire to reach the screen located on the left (blue line). The contagious radius was $2 \mathrm{~m}$.

situation are quite different. As the contagion stress $J$ increases, the bins become occupied earlier in time (higher slopes). For the most widely spread situation $(J=0.09)$ all the slices become occupied before the first 5 seconds, meaning that we may expect escaping pedestrians in any direction during most of the propagation process.

Fig. 9 represents the aforementioned three situations after $15 \mathrm{~s}$ since the (fake) bomber shout (see caption for details). These snapshots may be easily compared with the corresponding slice occupancy plot exhibited in Fig. 8.

Fig. 9a corresponds to the lowest contagion stress $(J=0.01)$. We can see an amorphous "branching" pattern for those pedestrians with fear (red circles). From the inspection of the whole process through an animation, we further noticed that these configuration could be classified into two types (see below).

A sharp "branching" profile is present in Fig. $9 \mathrm{~b}$ for $J=0.02$, although this category exhibits an extended number of pedestrians with fear. The highest contagion stress category $(J=0.09)$, instead, adopts a circular profile (see Fig. 9c).

Both "branching" profile (amorphous and sharp) observed for $J=0.01$ and $J=0.02$ (respectively) may be associated to the positive slopes in Fig. 8. Likewise, the circular profile for $J=0.09$ can be associated to the flat (blue) pattern therein. This suggests, once more, that two qualitative regimes may occur for the fear propagation in the crowd, as hypothesized in Section 5.1.1. Low contagion stresses correspond to the (qualitative) branch-like regime, while high contagion stress correspond to the (qualitative) circular-like regime. The snapshot in Fig. $2 \mathrm{~b}$ clearly shows a circular-like regime, as expected for the obtained experimental value of $J$.

The branching-like profile in Piazza San Carlo is not completely symmetric since the pedestrian's density is higher near the screen area (on the left of Figs. 2a and Fig. 5a) than in the opposite area. The pedestrians near the screen can not move away as easily as those in the opposite direction. Thus, the fear spreading near the screen occurs among almost static pedestrians, while the spreading on the opposite area occurs among moving pedestrians. Both situations, although similar in nature, produce an asymmetric branching. We labeled as passive branching the one near the screen, and active branching the one in the opposite direction.

It may be argued that since the $J=0.09$ pattern in Fig. 8 exhibits a positive slope at the very beginning of the fear propagation process and a vanishing slope a few seconds after (say, $5 \mathrm{~s}$ ), the association of branch-like to low $J$, and circular-like to high $J$, is somehow artificial. This is not true, as explained below.

We further binned the piazza into circular sectors around the (fake) bomber as shown in Fig. 8 (see caption for details). We carried out a similar analysis as in Fig. 8b, but for the sectors. Say, we computed the number of occupied sectors at each time-step. The results were similar as for the slices (not shown). This means that both (slices and sectors) behavioral patterns are strongly correlated (for any fixed $J$ ).

The speed of the radial "contagion" propagation, as computed from the number of occupied sectors (say, those with at least one anxious pedestrian), increases for increasing values of $J$ (not shown). Thus, a fast fear spreading is not only associated to high values of $J$, but also to circular-like profiles (for the piazza geometry) as observed above. The branch-like profiles, however, corresponds to a low contagion stress $J$ and therefore is it not expected to be found when high radial "contagion" speeds are present.

We may summarize the investigation so far as follows. The fear spreading dynamic may experience important (qualitative) changes according to the "efficiency" of the alerting process between neighboring pedestrians. This is expressed by the contagion stress parameter $J$. Our simulations shows that fear propagates weakly for low values of $J$. This produces a branch-like, slow fear spreading around the source of danger (for a simple geometry). However, if $J$ exceeds (approximately) 0.025 the fear spreads freely in a circular-like profile (for a simple geometry). The propagation also becomes faster.

It should be emphasized that $J \sim 0.025$ is an approximate threshold, but well formed circular-like profiles appear, in our simulations, for stresses above 0.03. Stresses beyond 0.04 exhibit similar profiles as those for $J \sim 0.04$. These results are valid for "contagion" radii between $2 \mathrm{~m}$ and $6 \mathrm{~m}$.

Notice that the explored range for contagion stresses $J$ does not exceed 0.1 . Thus, delays may appear during the fear propagation. 
In this context, it seems reasonable to attain similar results for "contagion" radii between $2 \mathrm{~m}$ and $6 \mathrm{~m}$, since the propagation velocity is bounded due to low spreading efficiencies.

The circular-like regime and the branch-like regime are consequence of two main factors: the radial escaping from the source of fear (see Section 4.2) and the fraction of pedestrians that switch to the anxious state.

For low values of $J$ there is a small fraction of pedestrians that gets anxious. We observed in the animations that these dodge the relaxed pedestrians in their way out. The neighboring pedestrians that gets anxious follow the free path left behind. Thus, a lane (one or more) of anxious pedestrians is formed across the crowd. The escaping pedestrians minimize in this way their efforts to get away from the source of fear. The branch-like pattern resembles this situation.

On the other hand, for the high contagion stress, the majority of the crowd gets anxious. Anxious pedestrians near the fake bomber escapes easily from his (her) due that also far away individuals run away too. So, they has not "resistance" from the crowd to escape from the source of fear. This is the reason for the circular-like pattern for high contagion stress.

Recall that the increase in the "inner stress" is the mechanism allowing the fear to spread among the crowd. The "emotional decay", however, can not be seems in the video of Piazza San Carlo (and in our simulations). This is because the emotional decay does not completely settle within the first $20 \mathrm{~s}$, although $\tau_{M}=10 \mathrm{~s}$. The examined time window is not long enough to show the anxious pedestrians to arrive to $v_{d}=0.5 \mathrm{~m} / \mathrm{s}$ (see Fig. 6). In this sense, the "emotional decay" seems not to play a relevant role within this time window (in the Piazza San Carlo only).

Finally, we want to remark that although the Piazza San Carlo (Turin) situation attains a highly symmetrical situation with a well localized source of danger (fake bomber), our first approach was to consider a circular room with the source of danger at the center (not shown). All relaxed pedestrians tried to stay at a fix position while individuals with fear were able to escape radially outward from the fake bomber. The results from this simple scenario agreed with the Piazza San Carlo situation for high values of $J$ (above 0.03). That is, the simulated crowd (with fear) adopted a circular pattern around the (fake bomber).

We will discuss in Section 5.2 a geometrically complex situation where either the "inner stress" and the "emotional decay" plays a relevant role.

\subsection{Charlottesville, virginia}

\subsubsection{Density contour}

We first computed the discretized density pattern at the beginning of the simulation process in order to compare it with the video pattern shown in Fig. 3b. We used the same cell size as in Fig. $3 \mathrm{~b}(1.5 \mathrm{~m} \times 1.5 \mathrm{~m})$. The corresponding contour density map can be seen in Fig. 10.

Figs. $3 \mathrm{~b}$ and Fig. 10 exhibit the same qualitative profiles. Also, the pedestrian occupancy per cell is similar on both figures. Notice that the middle of the region is occupied by two or more pedestrians per cell. The boundary cells, though, are occupied by a single pedestrian per cell in both figures. So, we may conclude that our initial configuration is qualitative and quantitative similar to the one in the video.

\subsubsection{The contagion stress parameter $J$}

Our next step was, as in the Turin case, to compute the mean number of pedestrians that switched to anxious in the first 15

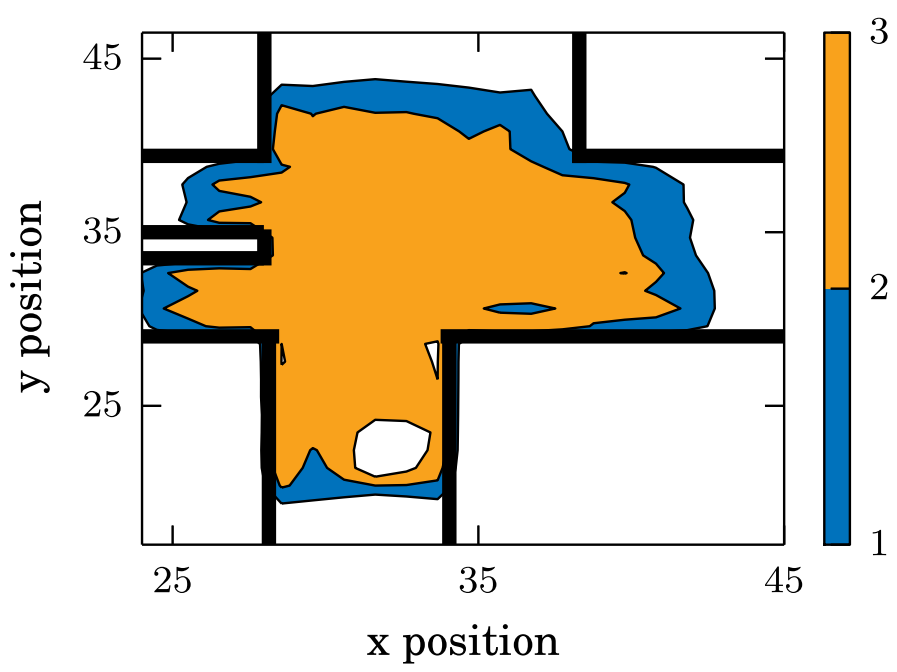

Fig. 10. Mean density contour lines computed from 90 processes. This density map corresponds to the initial state of the Charlottesville simulation. The scale bar on the right is expressed in pedestrians $/ \mathrm{m}^{2}$. The black lines represent the walls. The white circular shaped pattern at the bottom of the image corresponds to the car location (see Fig. 5b). The contour lines were computed on a squared grid of $1.5 \mathrm{~m} \times 1.5 \mathrm{~m}$ and then splined to get smooth curves. 


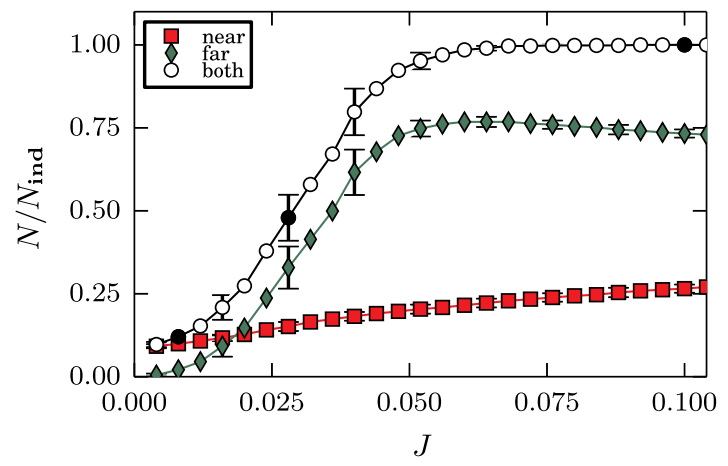

(a)

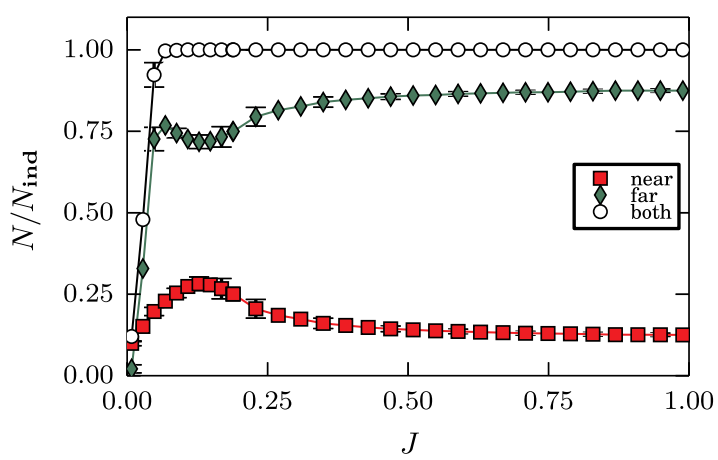

(b)

Fig. 11. Normalized number of pedestrians that switched to anxious in the first $15 \mathrm{~s}$ of the escaping process, as a function of the contagion stress $J$. Both figures (Fig. 11a and b) corresponds to the same analysis at different scale. $N$ is the number of anxious pedestrians. The plot is normalized with respect to the total number of individuals $\left(N_{\text {ind }}=600\right.$ ). The red squares corresponds to anxious pedestrians close to the car (less than $5 \mathrm{~m}$ ), while the green diamonds corresponds to those far away from the car (more than $5 \mathrm{~m}$ ). The white circles correspond to the (normalized) set of all individuals with fear. Results for $J=0.010,0.028$, and 0.1 are indicated in black color (and circle symbols) in (a). The contagious radius was $2 \mathrm{~m}$. Mean values were computed from 60 realizations. The error bars corresponds to $\pm \sigma$ (one standard deviation).

seconds of the escaping processes as a function of the contagion stress $(J)$. The results can be seen in Fig. 11 .

We observe that, likewise the Turin case, the total number of anxious pedestrians (circle symbols in Fig. 11) increases for increasing contagion stresses. From the comparison between Figs. 7 and Fig. 11a we may realize that both situations exhibit the same qualitative patterns for the total number of anxious pedestrians. However, the slope for the Charlottesville situation is somewhat lower with respect to the Turin situation (see Fig. 7).

In order to explain this slope discrepancy we computed, separately, the (mean) number of anxious pedestrians close to the car (i.e. source of fear) and those far away from the car. Recall from Section 4 that the former correspond to better informed pedestrians than the latter. The computation of the number of "near" anxious pedestrians actually include those pedestrians that gets anxious very close to the car (less than $1 \mathrm{~m}$ ).

We may recognize from Figs. $11 \mathrm{a}$ and $\mathrm{b}$ the same three qualitative categories mentioned in Section 5.1.1, according to the contagion stress value. Notice, however, that the anxious pedestrians settle to the relaxed state after 3 seconds (if near the car) or 20 seconds (if far away from the car). We will examine these regimes in the following sections.

\section{The low contagion stress regime}

For $J$ ranging between 0 to 0.02 , most of the pedestrians that get anxious are close to the car, while pedestrians far away from the car remain in a relaxed state. This means that fear does not spread homogeneously over all the crowd.

Recall from Section 4.1 that individuals located very close to the car (less than $1 \mathrm{~m}$ ) gets anxious immediately. So, as the car moves across the crowd, the fear propagates first over these nearby individuals. This explains why, in Fig. 11a, there is a small number of anxious pedestrians for extremely low contagion stresses $(J \sim 0)$.

Notice that this small group of anxious pedestrians represent the first source of fear inside the crowd (regardless of the car). As the susceptibility to fear emotions increase, their neighbors gets anxious. But, due to their rapid fear decay $\left(\tau_{M}=1.44 \mathrm{~s}\right.$ or $\left.t_{c}=3 \mathrm{~s}\right)$, their influence on the surrounding neighbors is low. This is the reason for the smooth increment of the near anxious pedestrians.

Besides, we can observe from Fig. 11a that the number of far away pedestrians (from the car) that gets anxious is not significant. This is because the pedestrians near the car get relaxed before reaching the far away area (say, more than $5 \mathrm{~m}$ away from the car). That is, if the number of "near" anxious pedestrians is low, while also relaxing quickly (i.e. 3 s), then the "probability" that fear reaches far away pedestrians from the car should be indeed very low.

\section{The intermediate contagion stress regime}

The fear spreading scenario changes if $J$ ranges between 0.02 and 0.05 . Along this interval, the total number of anxious pedestrians (white circles) increases abruptly. We can observe that this corresponds essentially to the increase in the amount of far away anxious pedestrians. Indeed, the number of near anxious pedestrians shown in Fig. 11a exhibits a smooth increment that cannot explain the abrupt increase of the total number of anxious individuals.

Notice that an increment in the number of anxious "far away" pedestrians becomes possible (at intermediate and high contagion stresses $J$ ) due to the significant time window that they spend surrounded by other "far away" anxious pedestrians (i.e. 20 seconds). Thus, the compound effect of high susceptibility to fear emotions and the long lasting time decays $\left(\tau_{M}=10 \mathrm{~s}\right.$ or $\left.t_{c}=20 \mathrm{~s}\right)$ explains the sharp increase in the number of anxious pedestrians. 


\section{The high contagion stress regime}

Finally, if the contagion stress becomes intense (say, above 0.05), most of the individuals gets anxious regardless of the precise value of $J$. Thus, as in the Turin situation, we may consider an approximate threshold for this regime around $J=0.07$. Fig. 11 b shows, however, that two noticeable behaviors appear whether the contagion stress is (roughly) below or above $J=0.2$ (despite the fact that the majority gets anxious).

Below $J=0.2$, the number of pedestrians that gets anxious near the car increases for increasing contagion stresses, while above this threshold the corresponding slope in Fig. 11b changes sign. The number of far away anxious pedestrians exhibit, though, a small "U" shape and a positive slope for $J \gg 0.2$ (see Fig. 11b).

The increase in the number of individuals that gets anxious near the car just below the threshold $J=0.2$ is a consequence of the increase in the susceptibility to fear emotions. But, above $J=0.2$, the situation is somewhat different. The contagion stress is so intense that fear propagates rapidly into the crowd. People standing as far as $5 \mathrm{~m}$ from the car may switch to an anxious state, and thus, they gets anxious before the car (i.e. the source of fear) approaches them. Our simulation movies confirm this phenomenon. We further realized that many of the anxious individuals located near the car and exhibited as red squares in Fig. $11 \mathrm{~b}$ for $J \leq 0.2$ may actually belong to the curve with green diamonds at extremely intense stresses $J \gg 0.2$.

The above research may be summarize as follows. We identified three scenarios according to the contagion stress $J$. If the susceptibility to fear emotions is low (below $J=0.02$ ), the fear spreads over a small group of pedestrians located very close to the car. In the case of an intermediate contagion stress ( $J$ between 0.02 and 0.05 ), the number of pedestrians that gets anxious far away from the car increases abruptly. Above $J=0.05$, the fear spreads over all the crowd.

The propagation velocity of the fear among the crowd is related to the contagion stress $(J)$. As the susceptibility to fear emotions increases, the fear spreading velocity also increases. So, if pedestrians are very susceptible to fear emotions, just a small number of individuals is capable of spreading fear over the whole crowd.

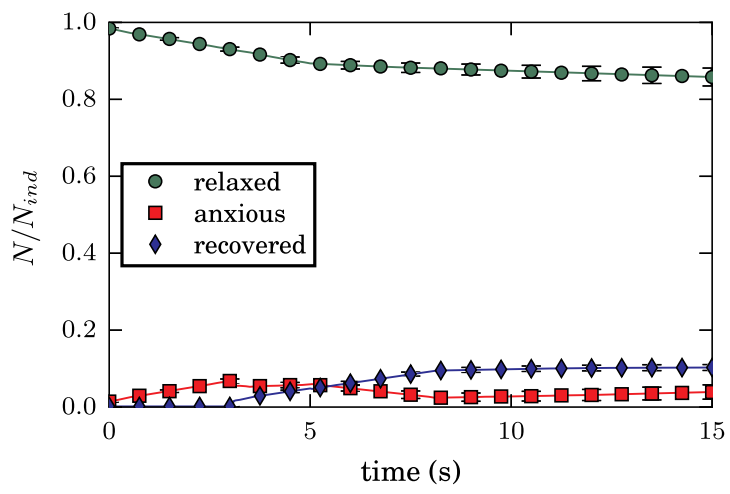

(a) $J=0.01$

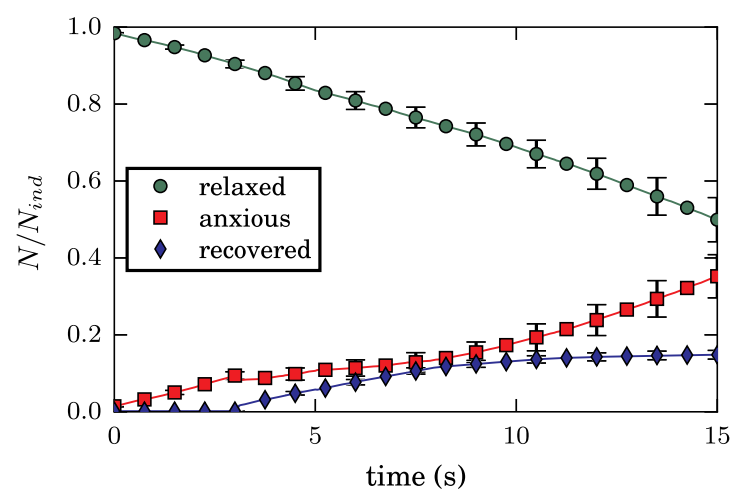

(b) $J=0.028$



(c) $J=0.1$

Fig. 12. Normalized number of pedestrians classify as relaxed, anxious and recovered, as a function of time $(J=0.01,0.028$ and 0.1$) . N$ is the number of pedestrian in each "emotional" state. The plot is normalized with respect to the total number of individuals $\left(N_{\text {ind }}=600\right)$. The green circles correspond to relaxed pedestrians, while the red squares correspond to anxious pedestrians (we computed jointly the number of anxious pedestrians close and far away from the car). The blue diamonds correspond to the recovered pedestrians (pedestrians that has been anxious, but actually are relaxed). Mean values were computed from 30 realizations. The error bars corresponds to $\pm \sigma$ (one standard deviation). 


\subsubsection{The contagion process along time}

In the previous Section, we computed the number of anxious pedestrians as a function of the contagion stress $J$. Here we focus on the behavior of these pedestrians as a function of time. The Fig. 12 shows the number of pedestrians in the relaxed, anxious or recovered state for $J=0.01,0.028$ and 0.1 . These situations corresponds to the qualitative categories mentioned in Section 5.2.2. That is, $J=0.01$ means a weak fear spreading, $J=0.028$ corresponds to an intermediate spreading and $J=0.1$ corresponds to a strong spreading (see caption of Fig. 12 for details).

According to Fig. 12, in all cases the number of relaxed pedestrians decreases monotonically during the escaping process. This accomplishes the fear spreading among the crowd. However, the slopes observed in Fig. 12 are quite different for each situation. The greater the contagion stress $J$, the sharper the slope. Since these slopes are related to the speed at which the fear propagates, Fig. 12 expresses the fact that the fear propagates faster as the pedestrians become more susceptible to fear emotions.

Besides, the behavior of the anxious individuals exhibits three major (qualitative) patters. For the $J=0.01$ case, the number of anxious pedestrians increases during the first 3 seconds. But after this initial period, it decreases smoothly, and finally reaches a stable value $(t>10 \mathrm{~s})$. This behavior can be explained with the help of Fig. 11a. The smooth decrease corresponds to the decay of the anxious pedestrians close to the car $\left(t_{c}=3 \mathrm{~s}\right)$. A few of them, however, may alert the far away pedestrians, "stabilizing" the amount of anxious individuals at the end of the propagation process.

The scenario changes for the $J=0.028$ and $J=0.1$ situations. Fig. $12 \mathrm{~b}$ and c show that, unlike the case $J=0.01$, the number of anxious pedestrians increases monotonically after the first 3 seconds. This phenomenon was already explained in Section 5.2.2. The contagion stress $J$ is strong enough to allow a continues growth of anxious people, sustained by the long stress decay $\left(t_{c}=20 s\right)$ of the far away pedestrians. Roughly speaking, the far away pedestrians dominate the scene because of the compound effect of high susceptibility, long lasting time decays, and many involved pedestrians.

Notice that for very strong contagion stresses (say, $J=0.1$ ) the number of anxious pedestrians reaches a maximum and holds after the first 10 seconds. At the same time, the number of pedestrians that gets anxious vanishes (see the relaxed curve in Fig. 12c). This is a stationary situation meaning that fear has already spread among the whole crowd. The video shows that the far away pedestrians (non-informed ones) continue escaping, despite the car stopped (see the anxious curve in Fig. 12c). The pedestrians close to the car switched to the relaxed state (labeled as "recovered" in Fig. 12).

\subsubsection{The morphology of the escaping pattern}

In Section 5.2.2 we computed the total number of anxious pedestrians as a function of the contagion stress $J$. Now, we examine the pedestrian's spatial distribution. We computed the Minkowski functionals (area and perimeter) for different contagion stresses $J$. The results are shown in Fig. 13.

The examined situations attain the same qualitative categories mentioned in Section 5.2.2. That is, $J=0.01$ for low fear spreading and $J=0.028$ for an intermediate spreading, and the two cases $(J=0.1$ and $J=0.3)$ for the highly intense situation. We also analyzed the limiting case $(J=1)$. No distinction was made at this point between relaxed or anxious pedestrians.

Recall from Section 2.5, that the area is the number of occupied cells by, at least, one pedestrian. Fig. 13a shows two qualitatively different patterns, one before the first 4 seconds and the other one after this time period. The former exhibits a slightly negative slope, while a positive slope can be seen in the latter (at least for a short time period).

The first 4 seconds in the fear propagation process correspond to the time period since the car strikes against the crowd until it stops. So, we may associate the decrease in the area with the movement of the pedestrians next to the car. The process animations actually show that these individuals group themselves as the car moves towards the crowd.

The slope of the area changes sign after the first 4 seconds, meaning an increase of the occupied area (see Fig. 13a). This corresponds, according to our animations (not shown), to pedestrians running away from each other. The greater the contagion stresses $J$, the sharper the slope. Since these slopes represent the escaping velocity, Fig. 13a expresses the fact that people may escape

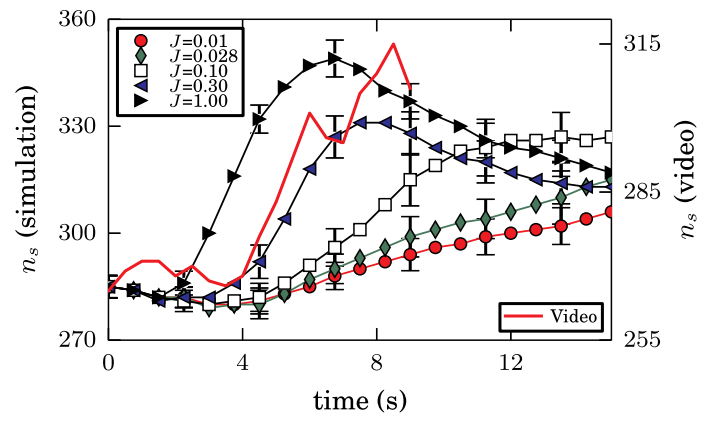

(a) Area.

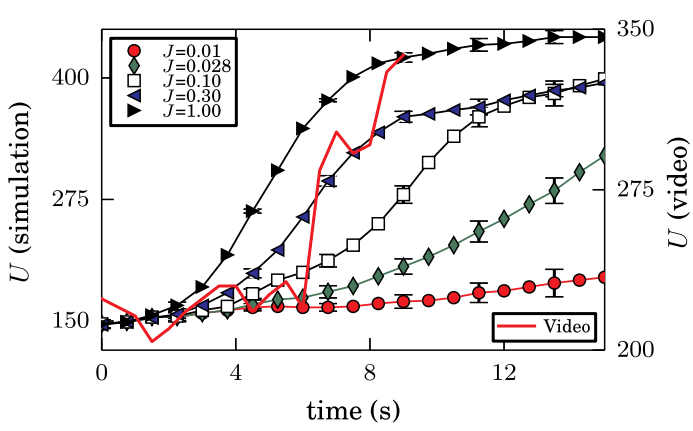

(b) Perimeter.

Fig. 13. Minkowski functionals corresponding to the (a) area $\left(n_{s}\right)$ and (b) perimeter $(U)$ (see Section 2.5 for more details). Each symbol corresponds to the Charlottesville simulation. The red line corresponds to the experimental data extracted from the Charlottesville incident. In both cases, the cell size was $1.5 \mathrm{~m} \times 1.5 \mathrm{~m}$. The duration of the Charlottesville video was 10 seconds. Mean values were computed from 90 realizations. The error bars corresponds to $\pm \sigma$ (one standard deviation). 


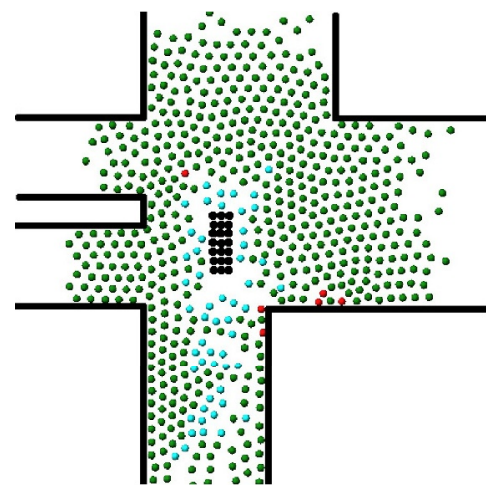

(a) $J=0.01$

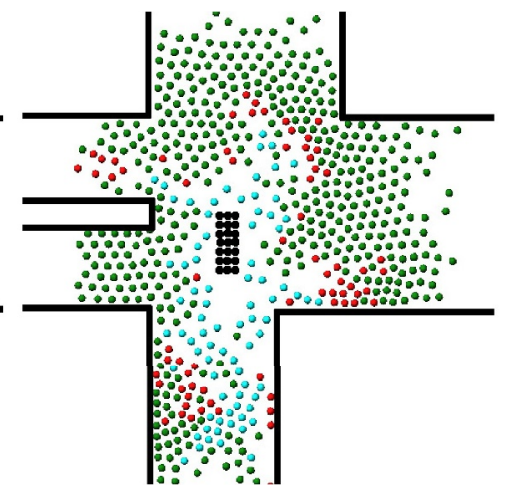

(b) $J=0.028$

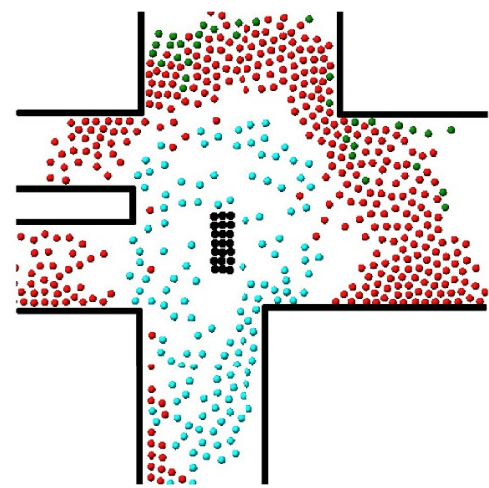

(c) $J=0.3$

Fig. 14. Snapshots of different escaping processes for low $(J=0.01)$, intermediate $(J=0.028)$ and high $(J=0.3)$ contagion stress in the first 10 seconds. The different colors of the circles represents the anxiety state of each pedestrian. Relaxed and anxious pedestrians are represents in green and red circles, respectively. The cyan circles represents the recovered pedestrians. That is, individuals that in the past were anxious but now are relaxed due their rapid fear decay $\left(t_{c}=3 \mathrm{~s}\right)$. The offending driver is represented by black circles. The solid lines represents the walls and the row of cars located on the middle left of the image (see Fig. 3).

faster as they become more susceptible to fear emotions (at least during this short time period).

Fig. 13b exhibits the results for the computed perimeter. This functional informs us on the length of the (supposed) boundary enclosing the crowd. Unlike the area, the perimeter appears as an increasing function of time (for the inspected values of $J$ ). Furthermore, as the susceptibility to fear emotions increases, the faster the perimeter widens (for a discussion on possible bias in the slopes of the curves see Appendix C).

The real life data included in Fig. 13 matches (qualitatively) the simulated patterns. Simulations corresponding to high contagion stresses appear to match better. The Minkowski functionals computed for $J=0.3$ exhibit the best matching patterns. Notice, however, that the y-scales of the experimental data and our simulations are not exactly the same (see Fig. 13). This is because of small discrepancies in the dimensions of the street crossing. Either the experimental and the simulation cells were set to $1.5 \mathrm{~m} \times 1.5 \mathrm{~m}$. But, the total number of cells did not match exactly due to quantifications errors and the corresponding perspective corrections.

We finally examined the process animations for low $(J=0.01)$, intermediate $(J=0.028)$ and high $(J=0.3)$ contagion stresses separately. The complementary snapshots are shown in Fig. 14, captured after 10 seconds from the beginning of the process. We chose this time interval in order to differentiate the three situations more easily (see caption for details).

Fig. 14a corresponds to the lowest contagion stress $(J=0.01)$. As already shown from Fig. 11a, only a small number of pedestrians gets anxious (due the low susceptibility to fear emotions). These pedestrians are colored in cyan in Fig. 14a (on-line version only), and correspond to people that was anxious and now recovered their relaxed state. No dramatic differences appear between the profiles shown in Figs. 5b and Fig. 14a. Thus, we may expect a smooth slope for the Minkowski functionals (see Fig. 13).

Fig. 14b shows a somewhat different scenario for $J=0.028$ (the intermediate contagion stress). We realize that an increasing number of pedestrians are now anxious. Many pedestrians that appeared as relaxed in Fig. 14a, have now become anxious because of the fear emotions from the individuals located near the car. However, the occupied area did not change significantly from Fig. 14a. The perimeter, instead, is expected to increase because of the voids left back by the anxious pedestrians inside the crowd (see the red circles in the on-line version of Fig. 14b).

The more stressing scenario is shown in Fig. 14c. The whole crowd gets anxious for $J=0.3$. This situation is comparable to the Turin incident (above $J=0.09$ ), despite the obvious geometrical differences. Thus, qualitatively speaking, Figs. 4 and Fig. 14c show "similar" crowd profiles.

A few conclusions can be outlined from the above analysis. As in the Turin situation, we found different scenarios according the pedestrian's susceptibility to fear emotions. For low contagion stresses, the fear spreads over a small group of pedestrians standing close to the source of fear (the car). As the contagion stress $(J)$ increases, the influence of these nearby individuals on their neighbors become more relevant. Thus, more pedestrians gets anxious. If $J$ is above 0.05 , the fear spreads over all the crowd.

Despite the fact that above $J=0.05$ all the pedestrians becomes anxious, there are two qualitatively different regimes, bounded by a threshold at (roughly) $J=0.2$. We found that below $J=0.2$, the role of the pedestrians near the source of fear (say, the better informed pedestrians) is a relevant one, since they are the mean for propagating fear deep inside the crowd. But, above $J=0.2$, the contagion stress is so intense that fear propagates rapidly into the crowd even though a minimum number of individuals near the car gets anxious.

\section{Conclusions}

We proposed a model in which fear spreads in a crowd according to a epidemiological approach. In our work we have taken as experimental input the information about two real fear-spreading events. 
The propagation of fear offered a challenge to the emotional mechanism operating on the pedestrians. We only included the "inner stress" and "stress decay" as the main processes triggered during a fear situation in the context of the Social Force Model. Although the simplicity of this model, we attained fairly good agreement with the real fear-spreading events.

We handled the coupling mechanism between individuals through the contagion stress parameter $J$. This parameter appears to be responsible for increasing the "inner stress" of the individuals. Our first achievement was getting a real (experimental) value for $J$. The value for the Piazza San Carlo event was $0.1 \pm 0.055$.

We further noticed through computer simulations that $J$ controls the "contagion" dynamics. The Piazza San Carlo event illustrates the dynamic arising for high values of $J$, where everyone moves away from the source of stress. However, this might not be the case for low values of $J$. Only a small number of pedestrians will escape from danger, although many will roughly stay at their current position. The whole impression will be like random "branches" (the pedestrians with fear) moving away from the source of danger. We actually concluded that $J \sim 0.03$ is roughly the limit between both dynamics.

Our simulations attained qualitatively correct profiles for the escaping crowd for both the Piazza San Carlo and the Charlottesville street crossing. But these profiles are geometry-dependent, and therefore, not a unique profile could be established for any value of $J$ at different incidents. We know (for now) that geometries similar to Piazza San Carlo may produce branch-like profiles $(J<0.03)$ or circular-like profiles $(J>0.03)$.

The "stress decay" depends on the nature of the source of fear (say, whether if it corresponds to a fake alert or not) and the amount of information that the pedestrians get from this source. That is, far away pedestrians from the igniting point of fear (fake bomber in the Turin situation, or the offending driver in the Charlottesville situation) may not have enough information on the nature of the incident, but nearby pedestrians may get a more precise picture of the incident. The clearer this picture becomes to them, the faster they are allowed to settle down, and thus, the shorter the characteristic decay time $\left(\tau_{M}\right)$.

We realized, however, that a shorter characteristic time actually prevents the fear from spreading. This was not the case at the Piazza San Carlo, since the fake bomber was not (directly) at the sight of the pedestrians (who where watching the football match). The Charlottesville incident, however, exhibited two groups of individuals, according to the available information. We noticed that the group near the source of danger attained a shorter characteristic time $\left(\tau_{M}=1.44 \mathrm{~s}\right)$ than the far away pedestrians $\left(\tau_{M}=10 \mathrm{~s}\right)$.

What we learned from the street crossing incident at Charlottesville is that the resulting pedestrian's dynamic is a consequence of the competing effects of the "inner stress" (increased by "contagion" stimuli) and the "stress decay". Both are essential issues for a trusty fear propagation model. The parameters $J$ and $\tau_{M}$ appear as the most relevant ones within our model.

The $J$ and $\tau_{M}$ parameters may not always be available because of poor recordings or missing data. We experienced this difficulty with the video of the Charlottesville incident. But the experimental geometrical functionals, like the area or the perimeter, allowed the estimate of $J$ by comparison with respect to simulated data $(J \sim 0.3)$.

We want to remark that different "contagion" radii (between $2 \mathrm{~m}$ and $6 \mathrm{~m}$ ) did not produce significant changes on our simulations. This was unexpected, and thus, we may speculate that "spontaneous" spreading out of the usual spreading" range may not produce dramatic changes, if the probability of "spontaneous" propagation is small.

\section{Acknowledgments}

C.O. Dorso is a main researcher of the National Scientific and Technical Research Council (spanish: Consejo Nacional de Investigaciones Científicas y Técnicas - CONICET), Argentina and full professor at Departamento de Física, Universidad de Buenos Aires. G.A. Frank is an assistant researcher of the CONICET, Argentina. F.E. Cornes is a PhD Student in Physics. This work was supported by the National Scientific and Technical Research Council grant Programación Científica 2018 (UBA-CYT) Number 20020170100628BA.

\section{Appendix A. The contagion efficiency}

This Section is intended as the formal support leading to the expression of the "contagion efficiency" (Eq. (7)), and the meaning of the "effective stress". We show that the chance for any pedestrian to switch to the anxious state may be computed from the number of anxious neighbors and his (her) susceptibility (expressed by $J$ ).

As a general overview, we first show how the probability of contagion (say, the "contagion efficiency" $\mathcal{P}_{n}$ ) follows a Binomial-like distribution for a fixed number of neighbors $n$ (under simplifying assumptions). This is expressed by Eq. (A.2). We then extend the computation of the "contagion efficiency" to an arbitrary number of neighbors, since the $n$ may vary from unity to a maximum $M$ within a "contagion radius". This is expressed by Eq. (A.4). We finally define the "effective stress" parameter $J$ (Eq. (A.7)).

\section{A1. The contagion efficiency for fixed $n$}

Any individual among the crowd may increase his (her) anxiety level if his (her) neighbors are with fear. This is actually the propagation mechanism for fear: one or more pedestrians express their fear, alerting the others of imminent danger. The latter may gets anxious and thus, a "probability" exists for getting with fear.

We hypothesize that the "probability to danger" (contagion efficiency) is the cumulative effect of the alerting neighbors. That is, if $k$ pedestrians among $n$ neighbors are expressing fear, then the contagion efficiency $\mathcal{P}_{n}$ of an individual is

$$
\mathcal{P}_{n}=p_{n}(1)+p_{n}(2)+\cdots+p_{n}(n)
$$


where $p_{n}(k)$ represents the contagion efficiency of $k=1,2, \ldots, n$ pedestrians (among $n$ neighbors) expressing fear. The distribution for $p_{n}(k)$ is a Binomial-like distribution if any neighbor expresses fear with fixed contagion efficiency $p$, regardless of the feelings of other neighbors. If the feelings of any neighbor (among $n$ pedestrians) is not completely independent of the other neighbors, $p_{n}(k)$ should be assessed as a Hypergeometric-like distribution.

For the purpose of simplicity we assume that the Binomial-like distribution is a valid approximation for the $p_{n}(k)$ computation. Consequently,

$$
\mathcal{P}_{n}=\sum_{k=1}^{n}\left(\begin{array}{l}
n \\
k
\end{array}\right) p^{k}(1-p)^{n-k}=1-(1-p)^{n}
$$

The mean value of neighbors expressing fear $\langle k\rangle$ is $n p$. Thus,

$$
\mathcal{P}_{n}=1-\left(1-\frac{\langle k\rangle}{n}\right)^{n}
$$

It is worth noting that this expression holds for a fix value of $n$. That is, the contagion efficiency $\mathcal{P}_{n}$ is conditional to the amount of neighboring individuals $n$.

\section{A2. The contagion efficiency for any number of neighbors}

As mentioned above, $\mathcal{P}_{n}$ stands for the contagion efficiency (the chance to switch to anxious state) if $n$ remains fixed within the "contagion" radius. This is actually not the case throughout the crowd, and $n$ may vary from unity to an expected maximum $M$ (say, $n=1,2, \ldots, M)$. Therefore, the overall contagion efficiency $\mathcal{P}$ is expected to be the efficiencies $\mathcal{P}_{n}$, but weighted by the probability of attaining exactly $n$ neighbors within the "contagion radius" $\pi_{n}$. The law of total probability reads

$$
\mathcal{P}=\sum_{n=1}^{M} \mathcal{P}_{n} \pi_{n}
$$

where $\pi_{n}$ means the probability that there are exactly $n$ neighbors surrounding the anxious pedestrian (within the "contagion radius"). Notice that the expression (A.4) neither includes the term for $n=0$, nor the terms above $M$. The situation $n=0$ is not considered here since it corresponds to a "spontaneous contagion" to danger. Recall from Section 2.3 that we are not considering "longer range" propagation, envisaged here as "spontaneous" spreading within the "contagion radius".

The situation $n>M$ corresponds to individuals out of the "contagion radius", and thus, not really capable of alerting of danger. The limiting value $M$ is supposed to be related to the "contagion radius" and the crowd packing density.

Recalling Eq. (A.3), the contagion efficiency $\mathcal{P}_{n}$ may be expanded as

$$
\mathcal{P}_{n}=1-\left(1-n p+\ldots+p^{n}\right)=p f_{n}(p)
$$

The function $f_{n}(p)$ stands for the summation

$$
f_{n}(p)=n-\frac{n(n-1)}{2} p+\ldots+p^{n-1}
$$

Each contributing terms in $f_{n}(p)$ may be envisaged as the alert to danger due to groups of individuals of increasing size (for a fixed number of neighbors $n$ ). Notice, however, that the expression (A.6) holds if the feelings between neighboring pedestrians are completely independent. Otherwise, the function $f_{n}(p)$ should be considered unknown.

The overall contagion efficiency reads

$$
\mathcal{P}=\sum_{n=1}^{M} \pi_{n} \frac{\langle k\rangle}{n} f_{n}(p) \simeq J\left\langle\frac{k}{n}\right\rangle
$$

where $J$ represents an effective stress for the propagation, since it expresses in some way the efficiency of the alerting process. That is, no fear propagation will occur for vanishing values of $J$, while the pedestrian susceptibility to fear emotions will become more likely as $J$ increases. The stress $J$ may depend, however, on the probability $p$. Appendix B shows that this dependency is weak enough to be omitted in a first order approach.

The fraction $\langle k / n\rangle$ corresponds to the mean fraction of neighbors expressing fear with respect to the total number of neighbors. This mean fraction is computed over all the possible number of neighbors, according to Eq. (A.7).

We should emphasized that the limiting value $\mathcal{P}=1$ may be hard to achieve since it demands $J=1$. The assumptions leading to the Binomial-like distribution, expressed by Eq. (A.2), may not be completely fulfilled for this case because strong correlations may appear between alerting pedestrians. Our investigation, however, fixed into experimental data attaining values of $J$ not bigger than $J=0.3$ (see Sections 5.2.4 and Appendix B).

\section{Appendix B. The sampling procedure for turin}

The effective stress $J$ may be evaluated from any real life situation. Details on the sampling procedure for the Turin incident at 
Table B.2

Data provided from the Turin video (see Section 3.1 for details). Samples were taken at $0.5 \mathrm{~s}$ time intervals. The second column shows the number of pedestrians $n_{p}$ that switched to the fear state at the corresponding time step. The third column exhibits the (mean) ratio between neighbors with fear with respect to the surrounding neighbors. The fourth column corresponds to the contagion efficiency $\mathcal{P}$ computed as a "no-replacement" procedure (see text). The last column corresponds to the contagion stress $J$ computed from the third and fourth columns. The total number of individuals was $N=131$.

\begin{tabular}{lllll}
\hline$t$ & $n_{p}$ & $\langle k / n\rangle$ & $n_{p} /\left(N-N_{p}\right)$ & $J$ \\
\hline 0.5 & 1 & 0.17 & 0.0077 & 0.0453 \\
1.0 & 1 & 0.20 & 0.0077 & 0.0385 \\
1.5 & 5 & 0.43 & 0.0391 & 0.0909 \\
2.0 & 5 & 0.42 & 0.0406 & 0.0967 \\
2.5 & 2 & 0.13 & 0.0169 & 0.1300 \\
3.0 & 4 & 0.55 & 0.0345 & 0.0627 \\
3.5 & 6 & 0.36 & 0.0536 & 0.1489 \\
4.0 & 13 & 0.64 & 0.1226 & 0.1916 \\
4.5 & 11 & 0.68 & 0.1219 & 0.2344 \\
5.0 & 10 & 0.52 & 0.3055 & 0.4849 \\
5.5 & 22 & 0.63 & 0.5800 & 0.6444 \\
6.0 & 29 & 0.90 & 0.7143 & 0.8117 \\
6.5 & 15 & 0.88 & \\
\hline
\end{tabular}

Piazza San Carlo are given in Section 3.1.

As a first step, we identified those individuals that switched to the "fear" state along the image sequence. We also identified the surrounding pedestrians for each anxious individual, and labeled them as neighboring individuals (regardless of their current anxiety state). For simplicity, we used the same profile (shown in Fig. 2c) throughout the image sequence.

The mean fraction $\langle k / n\rangle$ was obtained straight forward from this data. Table B.2 exhibits the corresponding results (see second column).

Notice that the surrounding pedestrians actually correspond to the most inner ring of pedestrians enclosing the anxious individual, but not the ones within a certain radius. This radius, however, can be estimated from the (mean) packing density of the crowd.

The anxious pedestrians at the border of the examined area of Piazza San Carlo (see Fig. 2c) are not included in Table B.2 since it was not possible to identify all of their surrounding pedestrians.

The fraction of the anxious pedestrians $n_{p}$ to the total number of individuals $N$ is a suitable estimate for the overall contagion efficiency $\mathcal{P}$. However, the sampling procedure should be done with care. The fact that we are able to sample only a limited portion of the crowd reduces the sampling space along time. This means that the "true" fraction of anxious pedestrians should be $n_{p} /\left(N-N_{p}\right)$, where $N_{p}$ corresponds to the number of individuals already with fear. This is known as a sampling "without replacement" procedure.

Notice that if $N_{p}$ is negligible with respect to $N$, the estimate $n_{p} / N$ (sampling "with replacement") should (approximately) match $n_{p} /\left(N-N_{p}\right.$ ) (sampling "without replacement"). This expresses that the sampling space is still wide enough for assuming an "infinite" crowd.

Fig. B.15 shows the effective stress $J$ computed as the ratio between $\mathcal{P}$ and $\langle k / n\rangle$. The contagion efficiency $\mathcal{P}$ was estimated either

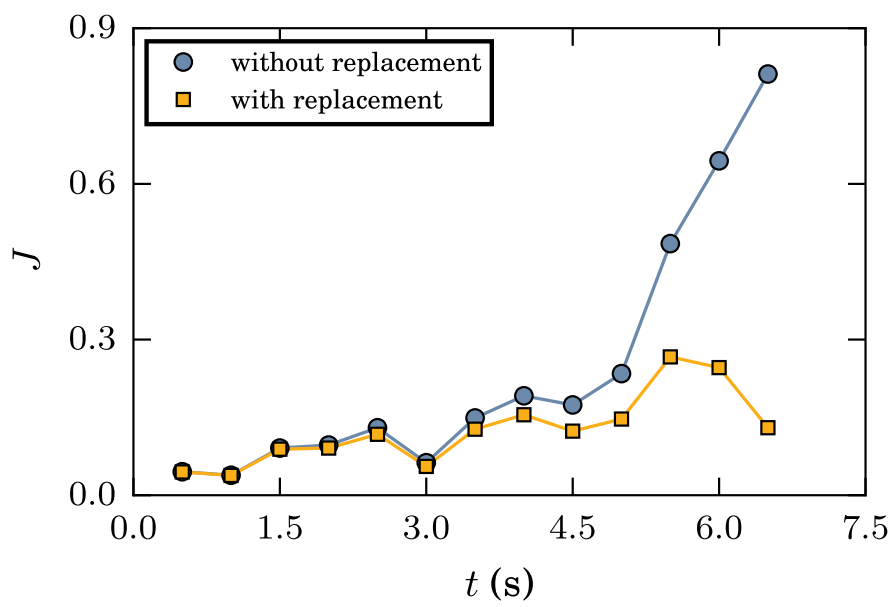

Fig. B.15. The contagion stress $J$ as a function of time $t$ in seconds (see text for details). The circle symbols (in blue color) correspond to the $J$ values computed from a crowd of $N=131$ individuals and a sampling procedure "without replacement" (see Table B.2 for details). The squared symbols correspond to the $J$ values computed from the same crowd, but following a sampling procedure "with replacement". The mean stress for $0.5 \mathrm{~s} \leq t \leq 4 \mathrm{~s}$ is $J=0.1 \pm 0.055$. 


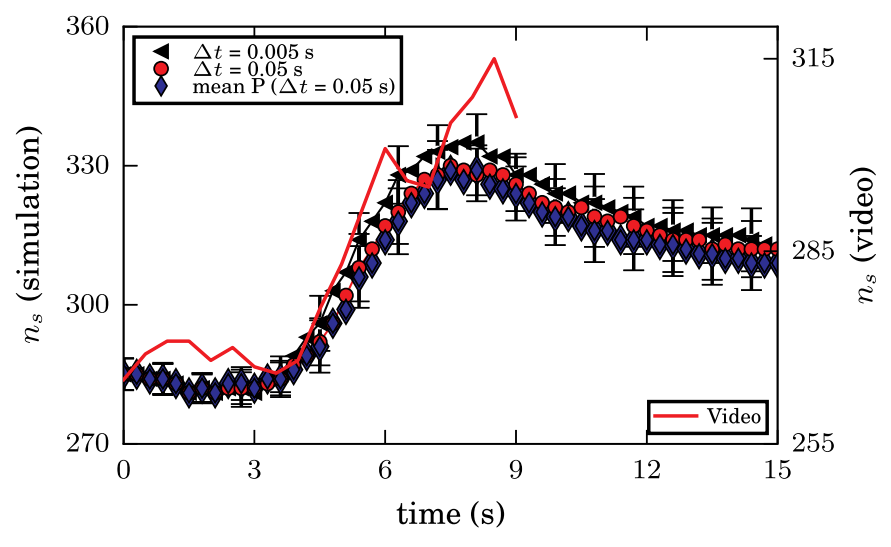

(a) Area

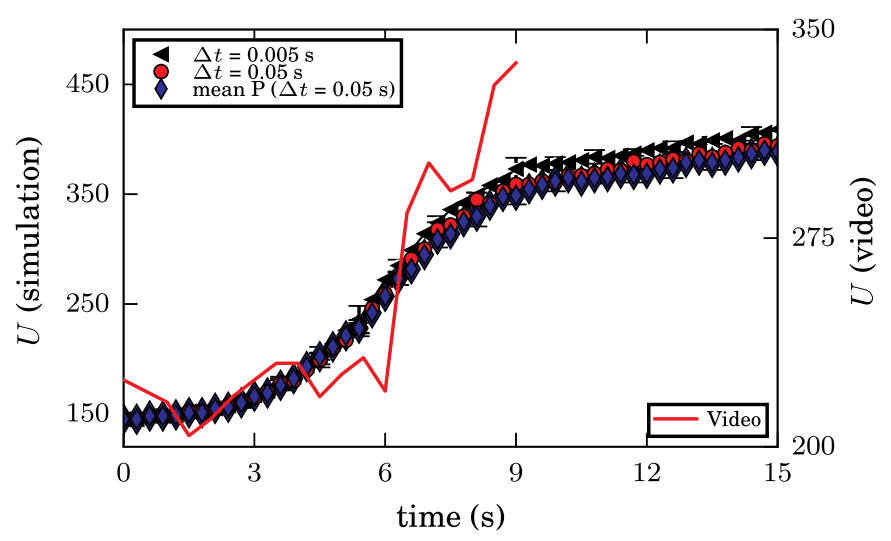

(b) Perimeter

Fig. C.16. Minkowski functionals corresponding to the area $\left(n_{s}\right)$ (a) and (b) perimeter $(U)$ for $J=0.3$ and $\tau_{i}=0.05$ s. The contagion efficiency $\mathcal{P}^{(i)}$ was computed every $\Delta t$ seconds ( $t$ in Eq. C.2). Each symbol correspond to the Charlottesville simulation, as follows: 4 corresponds to sampling subintervals of $0.005 \mathrm{~s}$ (without averaging), $\$$ corresponds to averaged values sampled at sub-intervals of $0.005 \mathrm{~s}$ (along the $0.05 \mathrm{~s}$ interval), and 0 corresponds to the usual interval $\tau_{i}=0.05 \mathrm{~s}$. The red line corresponds to the experimental data extracted from the Charlottesville incident. Mean values were computed from 30 realizations. The error bars corresponds to $\pm \sigma$ (one standard deviation).

as $n_{p} /\left(N-N_{p}\right)$ (i.e. without replacement) or $n_{p} / N$ (i.e. with replacement). It can be seen that both match for $t \leq 4 \mathrm{~s}$. That is, no boundary effects became relevant at this instance, and the crowd may be considered as "infinite".

The $J$ estimates exhibited in Fig. B.15 are not completely stationary along the interval $0.5 \mathrm{~s} \leq t \leq 4 \mathrm{~s}$. However, the increasing slope is not relevant for a first order approach. The mean value for the effective stress along this interval is $J=0.1 \pm 0.055$.

\section{Appendix C. The details of the integration scheme and time-scales}

Eqs. (11) and (A.7) requires the computation of $\langle k / n\rangle$. The $\langle\cdot\rangle$ operator stands for the mean value of the random variable $k / n$ at any chosen time-step. The process is assumed to evolve at regular time intervals (for computational reasons), although we are actually dealing with a (real-life) continuous stochastic process.

We mentioned in Section 4.2 that the "contagion" time interval (say, the "information time-step") was set to $\tau_{i}=0.05 \mathrm{~s}$. This is quite small with respect to the pedestrians relaxation time $\left(\tau_{i} \ll \tau\right)$. Thus, we may assume that the chances to switch to fear (per unit time) are somewhat uniform at the time scale of $\tau_{i}$. We consider this chances (per unit time) to be

$$
\lambda_{i}=\frac{J}{\tau_{i}}\left\langle\frac{k}{n}\right\rangle
$$

within this interval. The mean fraction appearing in the above expression is actually estimated as the observed fraction of fearing neighbors $k / n$.

Notice that the contagion efficiency $\mathcal{P}^{(i)}$ within any sub-interval can be computed from the following time integration 


$$
\mathcal{P}^{(i)}=\int_{0}^{t} \lambda_{i} d t \simeq \frac{J t}{\tau_{i}} \frac{k^{(i)}}{n}
$$

where the index $i$ indicates that the current sub-interval belongs to the ith-interval (lasting $\tau_{i}$ ). The $J t / \tau_{i}$ fraction may be envisaged as the contagion stress for that piece of interval. However, we do not intend to introduce it as a new magnitude for clarity reasons.

We checked our assumptions by computing the Minkowski functionals, as in the case of Fig. 13. But, we now introduce two differences: we sample $\mathcal{P}$ every $0.005 \mathrm{~s}$ (that is, every $\tau_{i} / 10$ ), and alternatively, we estimate $\langle k / n\rangle$ by averaging values sampled at the sub-intervals of $0.005 \mathrm{~s}$ (along the $0.05 \mathrm{~s}$ interval). The Fig. C.16 shows the corresponding Minkowski functionals (see caption for details). We also exhibit the results from the video analysis as a reference.

Notice that either changing the sampling frequency, or, replacing the single value estimates $k / n$ by the corresponding averages (of 10 data points within $\tau_{i}$ ) do not carry any significant bias. All the errors bars appear overlapped, meaning that no "artifacts" were introduced.

We should emphasize that the condition $\tau_{i} \ll \tau$ is crucial for attaining uniformity on the switching probabilities. The above integration scheme is no longer valid if this condition fails (say, if $\tau_{i} \sim \tau$ ). This is the reason for setting $\tau_{i}=0.05 \mathrm{~s}$, and consequently, referring the (simulated) contagion stress $J$ to this time scale.

Besides, the experimental value of $J$ extracted from the video analysis of the Turin incident was obtained out of the integration scheme (see Appendix B for details on the experimental method). As can be seen from Table B.2, this procedure does not assume uniformity (per unit time) but was checked to produce a regular experimental value of $J$, within the first 4 seconds of the evacuation.

\section{Supplementary material}

Supplementary material associated with this article can be found, in the online version, at 10.1016/j.simpat.2019.04.012

\section{References}

[1] S. Helivaara, H. Ehtamo, D. Helbing, T. Korhonen, Patient and impatient pedestrians in a spatial game for egress congestion, Phys. Rev. E 87 (2013) 012802.

[2] A. Schantz, H. Ehtamo, Spatial game in cellular automaton evacuation model, Phys. Rev. E 92 (2015) 012802.

[3] J.D. John, C. Cocking, S. Reicher, Everyone for themselves? a comparative study of crowd solidarity among emergency survivors, Br. J. Soc. Psychol. 48 (2012) 487-506.

[4] A. Mawson, Understanding mass panic and other collective responses to threat and disaster, Psychiatry 68 (2005) 95-113.

[5] D. Volchenkov, S. Sharoff, On the application of the dynamical systems theory to social modeling, in: P. Blanchard, R. Lima, L. Streit, R.V. Mendes (Eds.), The Sciences of Complexity: from Mathematics to Technology to a Sustainable World, Vol. CD-ROM, ZiF - Center for Interdisciplinary Research, 2001.

[6] H. Zhao, J. Jiang, R. Xu, Y. Ye, Sirs model of passengers' panic propagation under self-organization circumstance in the subway emergency, Math. Probl. Eng. 2014 (2014) 608315, https://doi.org/10.1155/2014/608315.

[7] L. Fu, W. Song, W. Lv, S. Lo, Simulation of emotional contagion using modified sir model: a cellular automaton approach, Phys. A 405 (2014) 380-391, https:// doi.org/10.1016/j.physa.2014.03.043. (Supplement C)

[8] G. Chen, H. Shen, G. Chen, T. Ye, X. Tang, N. Kerr, A new kinetic model to discuss the control of panic spreading in emergency, Phys. A 417 (2015) 345-357, https://doi.org/10.1016/j.physa.2014.09.055.

[9] X.H. Ta, B. Gaudou, D. Longin, T. Ho, Emotional contagion model for group evacuation simulation, Informatica 41 (2017) $169-182$.

[10] L. Fu, W. Song, W. Lv, X. Liu, S. Lo, Multi-grid simulation of counter flow pedestrian dynamics with emotion propagation, Simul. Model. Pract. Theory 60 (2016) 1-14, https://doi.org/10.1016/j.simpat.2015.09.007. (Supplement C)

[11] N. Pelechano, J. Allbeck, N. Badler, Controlling individual agents in high-density crowd simulation, Proc. 2007 ACM SIGGRAPH/Eurograph.Sympos. Comput. Animat. (2007) 99-108.

[12] A. Nicolas, S. Bouzat, M.N. Kuperman, Statistical fluctuations in pedestrian evacuation times and the effect of social contagion, Phys. Rev. E 94 (2016) 022313 , https://doi.org/10.1103/PhysRevE.94.022313.

[13] D. Helbing, I. Farkas, T. Vicsek, Simulating dynamical features of escape panic, Nature 407 (2000) 487-490.

[14] D. Helbing, P. Molnár, Social force model for pedestrian dynamics, Phys. Rev. E 51 (1995) 4282-4286.

[15] G.A. Frank, C.O. Dorso, Room evacuation in the presence of an obstacle, Phys. A 390 (2011) 172-185.

[16] I.M. Sticco, G.A. Frank, S. Cerrotta, C.O. Dorso, Room evacuation through two contiguous exits, Phys. A 474 (2017) $2135-2145$.

[17] D. Parisi, C.O. Dorso, Microscopic dynamics of pedestrian evacuation, Phys. A 354 (2005) 606-618.

[18] F.E. Cornes, G.A. Frank, C.O. Dorso, High pressures in room evacuation processes and a first approach to the dynamics around unconscious pedestrians, Phys. A 484 (2017) 282-298, https://doi.org/10.1016/j.physa.2017.05.013.

[19] C.O. Dorso, P.A.G. Molinelli, J.A. López, Topological characterization of neutron star crusts, Phys. Rev. C 86 (2012) 055805, https://doi.org/10.1103/PhysRevC. 86.055805 .

[20] K. Michielsen, H.D. Raedt, Integral-geometry morphological image analysis, Phys. Rep. 347 (6) (2001) 461-538, https://doi.org/10.1016/S0370-1573(00) 00106-X.

[21] W.S. Rasband, ImageJ, U. S. National Institutes of Health, Bethesda, Maryland, USA, https://imagej.nih.gov/ij/, 1997-2016, Imagej.

[22] G.A. Frank, C.O. Dorso, Evacuation under limited visibility, Int. J. Modern Phys. C 26 (2015) 1-18.

[23] I.M. Sticco, F.E. Cornes, G.A. Frank, C.O. Dorso, Beyond the faster-is-slower effect, Phys. Rev. E 96 (2017) 052303, https://doi.org/10.1103/PhysRevE.96. 052303.

[24] S. Plimpton, Fast parallel algorithms for short-range molecular dynamics, J. Comput. Phys. 117 (1) (1995) 1-19, https://doi.org/10.1006/jcph.1995.1039.

[25] D. Oberhagemann, S. Sharoff, Static and dynamic crowd densities at major public events, 2012, Technical Report. 